\title{
Ci antagonizes Hippo signaling in the somatic cells of the ovary to drive germline stem cell differentiation
}

Chaoyi $\mathrm{Li}^{1,}$, Lijuan Kan ${ }^{1, ~ *}$, Yan Chen ${ }^{2, ~ *}$, Xiudeng Zheng ${ }^{3}$, Weini $\mathrm{Li}^{1}$, Wenxin Zhang ${ }^{1}$, Lei $\mathrm{Cao}^{2}$, Xiaohui Lin ${ }^{2}$, Shanming $\mathrm{Ji}^{1}$, Shoujun Huang ${ }^{1}$, Guoqiang Zhang ${ }^{1}$, Xiaohui $\mathrm{Liu}^{2}$, Yi Tao ${ }^{3}$, Shian $\mathrm{Wu}^{2}$, Dahua Chen ${ }^{1}$

${ }^{I}$ State Key Laboratory of Reproductive Biology, Institute of Zoology, Chinese Academy of Sciences, Datun Road, Chaoyang, Beijing 100101, China; ${ }^{2}$ State Key Laboratory of Medicinal Chemical Biology and College of Life Sciences, Nankai University, Tianjin 300071, China; ${ }^{3}$ Centre for Computational and Evolutionary Biology, Institute of Zoology, Chinese Academy of Sciences, Beijing 100101, China

Many stem cell populations are tightly regulated by their local microenvironment (niche), which comprises distinct types of stromal cells. However, little is known about mechanisms by which niche subgroups coordinately determine the stem cell fate. Here we identify that Yki, the key Hippo pathway component, is essential for escort cell (EC) function in promoting germline differentiation in Drosophila ovary. We found that Hedgehog (Hh) signals emanating primarily from cap cells support the function of ECs, where Cubitus interruptus (Ci), the Hh signaling effector, acts to inhibit Hippo kinase cascade activity. Mechanistically, we found that Ci competitively interacts with Hpo and impairs the Hpo-Wts signaling complex formation, thereby promoting Yki nuclear localization. The actions of Ci ensure effective Yki signaling to antagonize $\mathrm{Sd} / \mathrm{Tgi} / \mathrm{Vg}$-mediated default repression in ECs. This study uncovers a mechanism explaining how subgroups of niche cells coordinate to determine the stem cell fate via Hh-Hippo signaling crosstalk, and enhances our understanding of mechanistic regulations of the oncogenic Yki/YAP signaling.

Keywords: Hippo; Yki; Hedgehog; Ci; Drosophila ovary; escort cell

Cell Research (2015) 25:1152-1170. doi:10.1038/cr.2015.114; published online 25 September 2015

\section{Introduction}

Stem cells can divide asymmetrically to self-renew and to generate differentiating daughter cells [1]. In this paradigm, an anterior germline stem cell (GSC) and a posterior cystoblast (CB) cell are produced in Drosophila ovaries [2, 3] (Figure 1A). The inherent asymmetry of GSC division depends on a microenvironment (or called niche) maintained by signals secreted from three types of somatic stromal cells, including terminal filament (TF) cells, cap cells (CpCs), and escort cells (ECs, also called inner germarium sheath (IGS) cells) [4-6]. Among these cells, CpCs directly contact GSCs in the tip of the ger-

\footnotetext{
*These three authors contributed equally to this work. Correspondence: Dahua Chen ${ }^{\mathrm{a}}$, Shian $\mathrm{Wu}^{\mathrm{b}}$

${ }^{a}$ E-mail: chendh@ioz.ac.cn

bE-mail:wusa@nankai.edu.cn

Received 30 April 2015; revised 2 August 2015; accepted 6 August 2015; published online 25 September 2015
}

marium, a functional unit that is divided into four regions (1, 2a, 2b, and 3; Figure 1A). CpCs can secrete decapentaplegic (Dpp, Drosophila BMP2/4) ligands that bind to a heterodimeric receptor comprising thickveins (Tkv) and Punt to activate the Smad signaling in GSCs and thus repress the transcription of bag of marbles (bam), which encodes an obligatory $\mathrm{CB}$ differentiation factor [7-12]. In contrast, ECs reside on the surface of regions 1 and $2 \mathrm{a}$ (Figure 1A) and associate with differentiating CBs and cysts to support normal germ cell differentiation $[5,6]$. However, the molecular mechanisms by which ECs support germ cell differentiation and the way in which ECs coordinate with $\mathrm{CpCs}$ to balance stem cell self-renewal and differentiation are poorly understood.

The Hippo signaling pathway has been shown to play evolutionarily conserved roles in controlling organ size and cell fate determination during development in both Drosophila and mammals [13-18]. The Hippo signaling pathway features a kinase cascade comprising the Ste20like kinase Hippo (Hpo), the nuclear Dbf-2-related (NDR) 

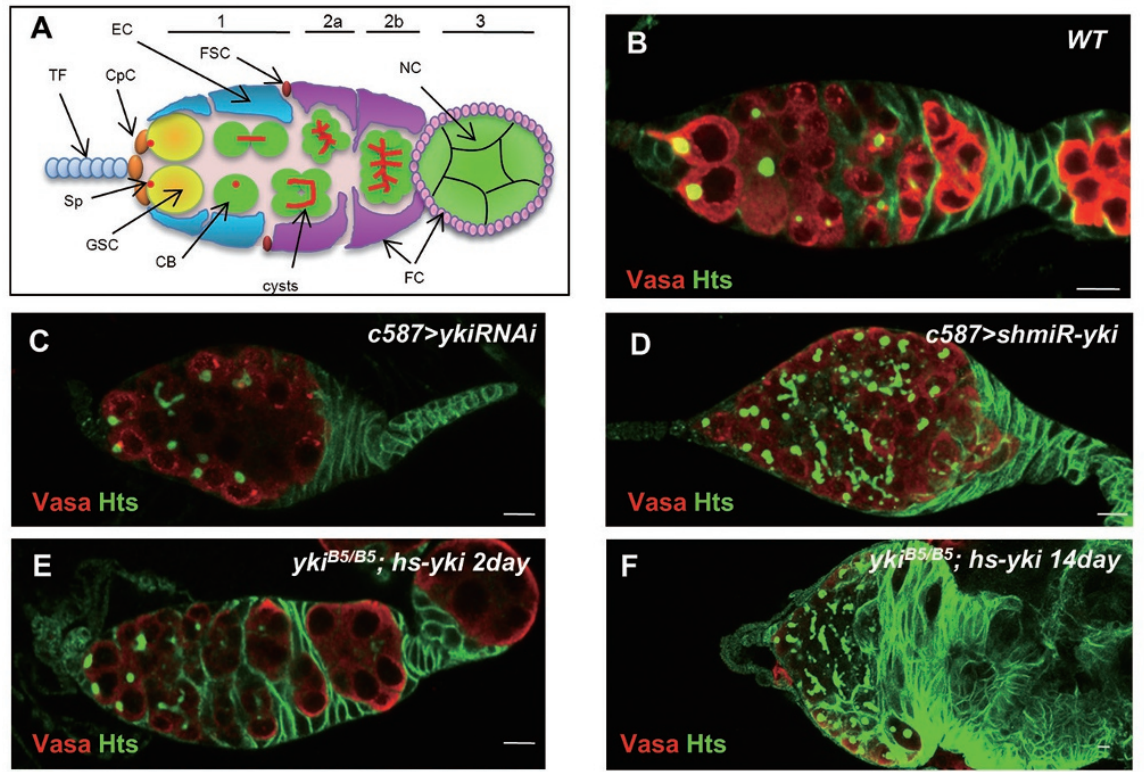

\section{G}

H 2-days

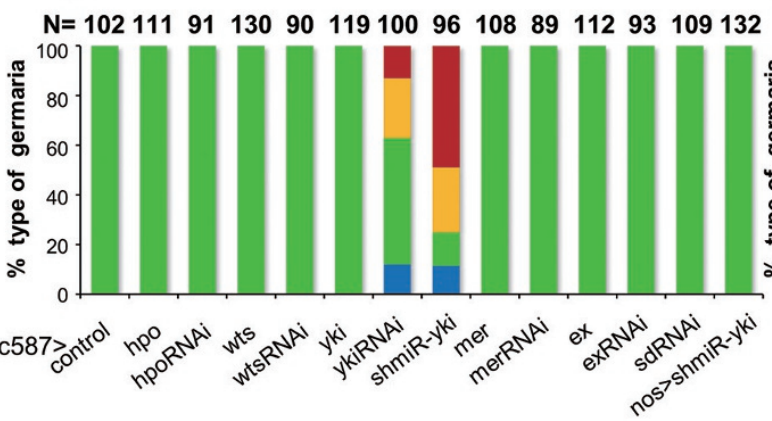

$\mathrm{N}=111104$

5-days 14-days
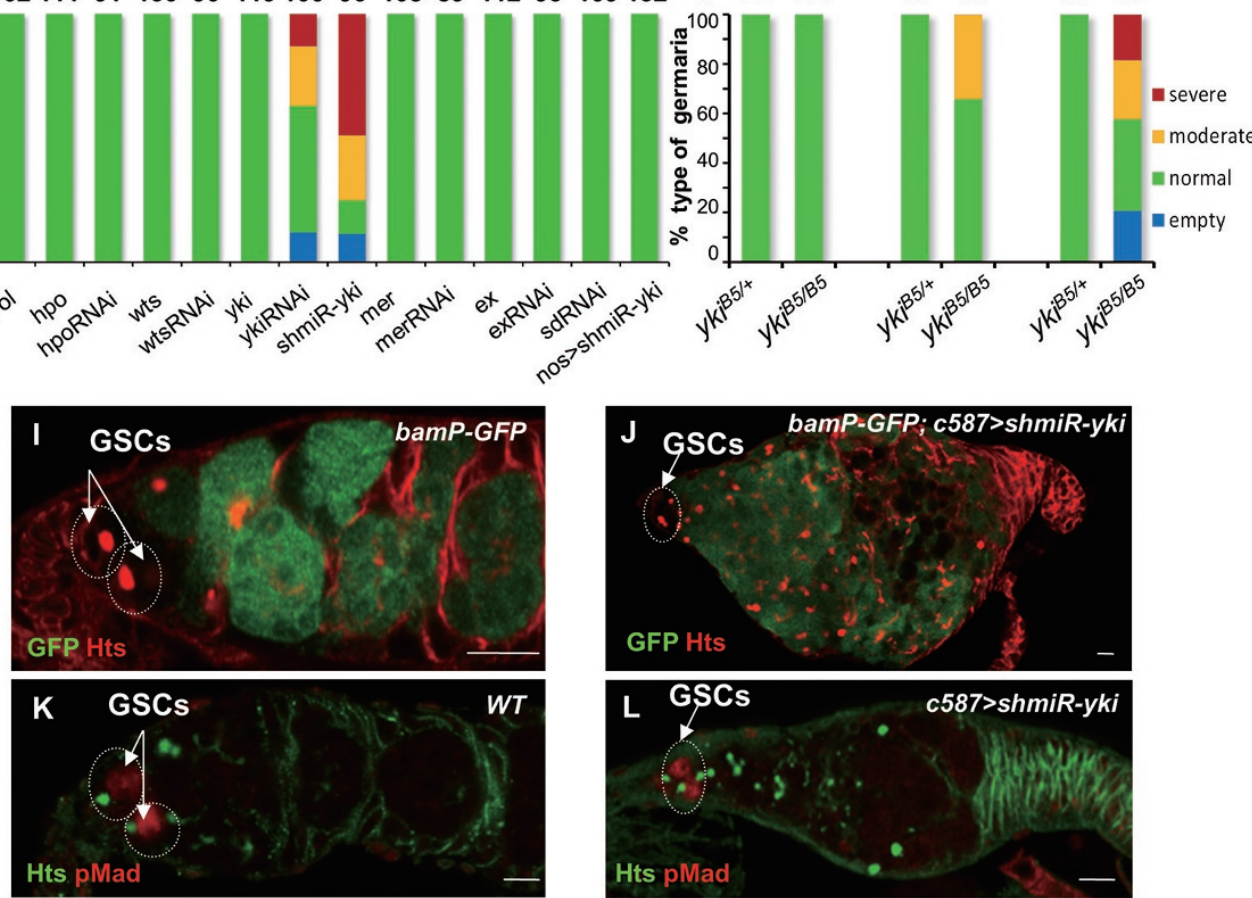

Figure 1 Yki acts as a somatic factor to promote early germ cell differentiation. (A) Schematic diagram of the germarium, with different cell types and organelles indicated as follows: terminal filament (TF), cap cell (CpC), escort cell (EC), germline stem cell (GSC), cystoblast (CB), follicle stem cell (FSC), follicle cell (FC), cyst (differentiated germ cell with extended or branched fusomes), and spectrosomes (Sp). (B-D) Ovaries from wild-type $w^{1118}$ (WT, B), c587; P\{uas-ykiRNAl\} (C) and c587; $\mathrm{P}\{$ uasP-shmiR-yki\} (D) were stained with anti-Hts (green) and anti-Vasa (red) antibodies. (E-F) Ovaries from the heat shock treatment-rescued females $y k^{B 5 / B 5} ; \mathrm{P}\{h s-y k i\}$ on day $2(\mathrm{E})$ and day $14(\mathrm{~F})$ post eclosion were stained with anti-Hts (green) and anti-Vasa (red) antibodies. Homozygous $y k i^{B 5 / B 5}$ mutants were lethal and were rescued by $\mathrm{P}\{h s-y k l\}$ with heat shock treatment every two days until the adult stage. (G) Quantification of the germarium phenotypes in ovaries with overexpression or knockdown of Hippo pathway components in ECs. The c587-gal4 was used as control. $(\mathbf{H})$ Quantification of the germarium phenotypes in ovaries from homozygous $y k i^{B 5 / B 5}$ mutants rescued by $\mathrm{P}\{h s-y k i\}$ with heat shock treatment until the adult stage. $\mathrm{P}\{h s-$ $y k l\} ; y k i^{B 5} / C y o$ was used as control. (I-J) Ovaries from P $\{$ bamP-GFP $\}$ (I) and P $\{$ bamP-GFP $/ c 587 ; \mathrm{P}\{$ uasP-shmiR-ykl\} (J) were stained with anti-Hts (red) and anti-GFP (green). (K-L) Ovaries from wild-type $w^{1118}$ (WT, K) and c587; P\{uasP-shmiR-yki\} (L) females were stained with anti-Hts (green) and anti-pMad (red). Scale bar, $10 \mu \mathrm{m}$. 
family kinase Warts (Wts), and their scaffold proteins, Salvador (Sav) and Mob as tumor suppressor (Mats). Hpo phosphorylates and activates Wts, which in turn phosphorylates the transcriptional co-activator Yorkie (Yki), leading to its cytoplasmic retention and inactivation $[13,15,19-21]$. In the absence of the upstream kinase cascade signaling, Yki is therefore released from the control of Hippo signaling and translocates into the nucleus, where it binds to the transcription factor, Scalloped (Sd), to activate downstream target genes including diap1, cyclin E and bantam, thereby promoting cell proliferation and inhibiting cell apoptosis [21-23]. Recently, an elegant study demonstrated that the primary role of Yki in normal tissue growth is to antagonize Sd-mediated transcriptional repression, where Tgi, a Tondu-domain-containing protein, functions as an Sd-associating factor to suppress the expression of Yki target genes [24]. In this default repression model, low-level Yki activation in the nucleus reverses Sd-mediated default repression, thus enabling normal tissue growth. In contrast, activation of the Hippo pathway turns off the nuclear functions of Yki and thus maintains Sd-mediated default transcriptional repression, which precludes cell growth [24, 25]. Thus, balanced regulation of Yki activation and Sd-mediated default repression is critical for organ size control during development, and is possibly important in other biological processes such as stem cell regulation and tumorigenesis $[13,20]$.

During development, cell proliferation and differentiation are governed by multiple signaling pathways that constitute a complex gene regulatory network that enables maximal plasticity and versatility of signal interpretation in response to environmental and developmental cues [26, 27]. In Drosophila ovary, the maintenance of GSCs and the differentiation of early germ cells largely depend on their surrounding somatic niche cells, which secrete multiple signaling ligands $[5,9,28]$. This organization raises the possibility that distinct types of somatic niche cells act cooperatively to form an integrative signaling network that exerts control over somatic niche function and regulates proper germline development.

In this study, we identify a novel role of Yki signaling in controlling the function of ECs by antagonizing Sd-mediated default repression, thus allowing the proper germline development. Interestingly, we found that Hedgehog ( $\mathrm{Hh}$ ) primarily released from $\mathrm{CpCs}$ activates its downstream signaling in ECs, where activation of the Hh signaling effector Cubitus interruptus (Ci) supports Yki signaling by antagonizing the upstream Hpo activity. Mechanistically, we found that $\mathrm{Ci}$ physically interacts with Hpo and Yki, and thus releases Yki from the inhibitory effect mediated by the upstream Hpo-Wts kinase cascade signaling. Moreover, the activation of $\mathrm{Ci}$ ensures an effective Yki signaling that antagonizes Sd-mediated default repression in ECs, thereby maintaining early germ cell differentiation.

\section{Results}

Yki acts as a somatic factor to promote early germ cell differentiation

Previous mosaic clonal studies have suggested that the Hippo pathway is not intrinsically required for GSC maintenance and early germline development $[29,30]$. However, since our immunostaining assay showed that the expression of Hpo and Yki, two key components of the Hippo pathway, was detectable in somatic cells in germaria (Supplementary information, Figure S1A-S1B), we sought to determine whether the Hippo pathway functions in ECs to control germ cell development. To do so, we employed the UAS-GAL4 system to overexpress hpo, wts, merlin (mer), expanded (ex) and yki (Supplementary information, Data S1) using the c587-gal4 driver that is mainly active in ECs (Supplementary information, Figure S1C). Immunostaining assays showed that individually overexpressing these Hippo components in ECs led to no detectable germarium phenotype (Figure 1B and Supplementary information, Figure S1D-S1H).

We then performed an RNAi assay to individually knock down the Hippo components in ECs. As shown in Supplementary information, Figure S1I-S1M, knockdown of hpo, wts, mer, sd, or ex did not affect germline development in germaria. However, we were surprised to find that knockdown of $y k i$ in ECs resulted in a tumorous germarium phenotype. As shown in Figure 1C and $1 \mathrm{G}$, a significant number of GSC/CB-like cells carrying spectrosomes were induced in $y k i$-knockdown germaria. Of note, $y k i$-knockdown germaria could be divided into three types, normal, moderate and severe types, although most of them belong to the "severe" type (Figure 2A$2 \mathrm{C}$ ). In addition, differentiated cysts with branched fusomes were also observed in $y k i$-knockdown germaria (Figure 1C). These findings raise a possibility that Yki might acts as a somatic regulator to promote early germ cell differentiation and to maintain a proper germ cell lineage in ovaries.

We next sought to validate this observation by generating the transgenic line $\mathrm{P}\{u a s P-s h m i R-y k i\}$ that carries artificial miRNAs targeting yki [31]. As shown in Figure $1 \mathrm{D}, 1 \mathrm{G}$ and Supplementary information, Figure S1N, expression of artificial miRNAs against $y k i$ driven by $c 587$ gal4, but not by the GSC-specific nosP-gal4:vp16 driver, produced a strong tumorous germarium filled with many GSC/CB-like cells and cysts. To further confirm the 
above observations, we performed a rescue experiment using a transgenic line, $\mathrm{P}\{h s-y k i\}$, in which a coding sequence for $y k i$ was placed under the control of the heatshock promoter. The $y k i$ homozygous mutants are lethal at the larval stage, but survived to adulthood when the $\mathrm{P}\{h s-y k i\}$ transgene was introduced and activated by a 60 -min heat-shock pulse at $38{ }^{\circ} \mathrm{C}$ every 2 days. Thus, by immediately withdrawing the heat-shock treatment after adult eclosion, the animals became progressively Yki-deficient as the protein decayed. Most germaria from 2-day-old yki mutant flies showed no significant phenotypic differences from wild-type controls in germarium, although a follicle cell defect was observed in some egg chambers in $y k i$-knockdown ovaries, compared with wild-type control (Figure 1E, 1H and Supplementary information, Figure S1O). By contrast, the 14-day-old yki mutant germaria exhibited a striking tumorous germarium phenotype (Figure $1 \mathrm{~F}$ and $1 \mathrm{H}$ ). Collectively, these findings support a notion that Yki acts as a somatic regulator in ECs to promote early germ cell differentiation.

\section{Yki regulates EC function and germ cell differentiation in a Dpp-independent manner}

We next attempted to explore the molecular basis of how loss of $y k i$ produces tumorous germarium phenotype. In Drosophila ovary, it was well documented that BMP/Dpp signaling represses the transcription of bam in GSCs, and that overexpression of Dpp leads to the formation of tumorous germaria that contain many $\mathrm{GSC} /$ CB-like cells expressing phosphorylated Mad (pMad) but not Bam [7, 9, 32]. Given that knockdown of $y k i$ in ECs also results in ectopic GSC/CB-like cells, and that Yki/ YAP was reported to regulate other signaling pathways including TGF- $\beta$ /BMP pathways via the non-canonical Hippo pathway [26], we therefore asked whether loss of $y k i$ induces ectopic GSC/CB-like cells in a BMP/Dpp signaling-dependent manner. Immunostaining assays showed that spectrosome-containing cells in $y k i$-knockdown tumorous germaria were bam-GFP- and Bam protein-positive but pMad-negative (Figure 1I-1L and Supplementary information, Figure S1Q-S1R). Because bam-GFP and pMad are commonly used as Dpp-responsive reporters in germ cells, our findings suggest that Yki acts in ECs to maintain germ cell differentiation in a manner largely independent of Dpp signaling. To gain more evidence to support our conclusion, we generated a new transgenic line, dadP-GFP, in which GFP was placed under the $d a d$ promoter since $d a d$ is a typical target gene of Dpp signaling in Drosophila. As expected, dadP-GFP is highly expressed in GSCs, and weakly expressed in a portion of $\mathrm{CB}$ cells in wild-type ovaries. However, in c587>shmir-yki ovaries, dadP-GFP expres- sion was restricted in the anterior position (GSCs and some $\mathrm{CBs}$ ), and most of spectrosome-containing cells were GFP-negative (Supplementary information, Figure S1S-S1T).

\section{Yki maintains EC function through the canonical Hippo} pathway

Of note, we found that while overexpression of $\mathrm{Sd}$ by $c 587$-gal4 was lethal, knockdown of $s d$ driven by c587-gal4 produced no apparent germarium phenotype (Supplementary information, Figure S2A), and these animals were fertile. Given the recently proposed default repression model [24], we tested whether depletion of $s d$ could reverse $y k i$ knockdown-induced tumorous germarium phenotype, and found that it indeed did (Figure 2D2E). Notably, we found that while $y k i$ knockdown in ECs caused female sterility, $y k i$ and $s d$ double-knockdown females were fertile and exhibited normal germline development (Supplementary information, Figure S2B-S2C). Thus, these assays argue that Yki maintains EC niche function via the canonical Hippo pathway.

\section{The Tondu-domain-containing proteins suppress germ} cell differentiation via $\mathrm{Sd}$

Our genetic tests suggest that Sd suppresses germ cell differentiation by acting as a default repressor in ECs, whereas Yki antagonizes the default repressing activity of $\mathrm{Sd}$, thus ensuring proper germ cell differentiation. To further test this, we took advantage of the temperature-dependent activity of the Gal4 to spatio-temporally overexpress Sd in ECs, since overexpression of Sd usually causes animal lethality under normal temperature $\left(25^{\circ} \mathrm{C}\right)$. Following the method described previously [33], crosses were performed and the resulting progenies were initially raised at $18{ }^{\circ} \mathrm{C}$ until adult eclosion, at which point they were shifted to $29^{\circ} \mathrm{C}$. As shown in Supplementary information, Figure S2D, overexpression of Sd in ECs using c587-gal4 did not produce apparent germarium phenotype. These findings suggest that $\mathrm{Sd}$ acts as a permissive regulatory component in the default repression complex in ECs.

We next sought to search for the instructive factor(s) in the default repression complex in ECs. Recent studies have identified Tgi, a Tondu-domain-containing protein, as an $\mathrm{Sd}$ cofactor in the default repressing complex to regulate tissue growth in Drosophila imaginal discs [24, $25]$. We followed the above protocol to test whether Tgi contributes to the Sd-mediated default repression in ECs. As shown in Figure 2F-2G and Supplementary information, Figure S2E, tgi knockdown driven by c587 suppressed the tumorous germarium phenotype induced by yki knockdown in ECs, and rescued fertility of females 


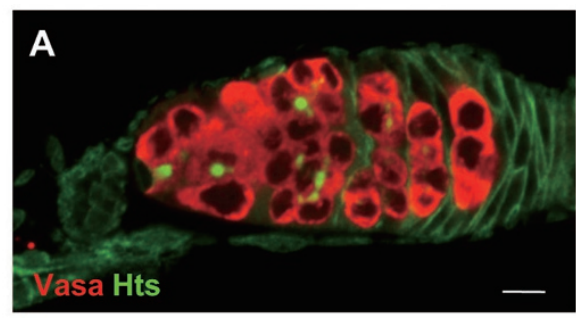

Normal

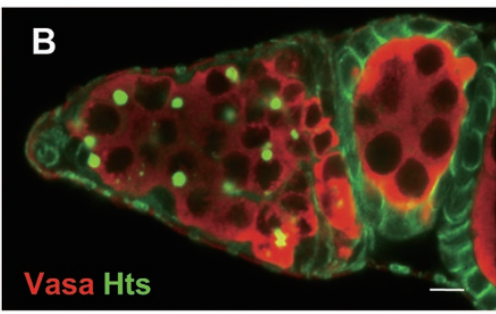

Moderate

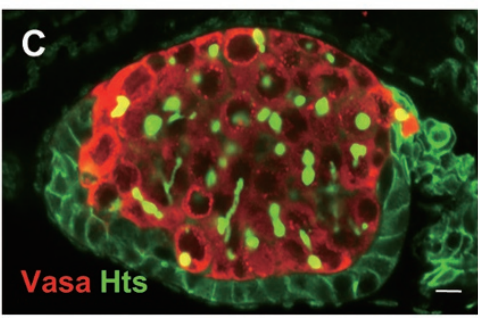

Severe

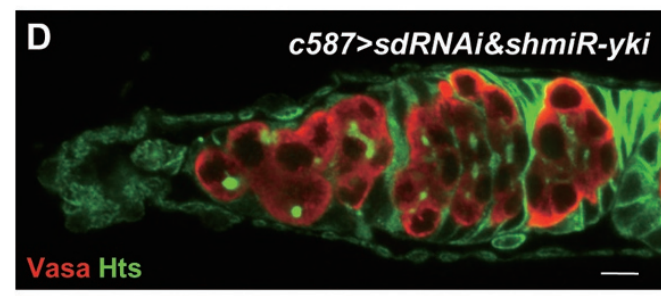

E
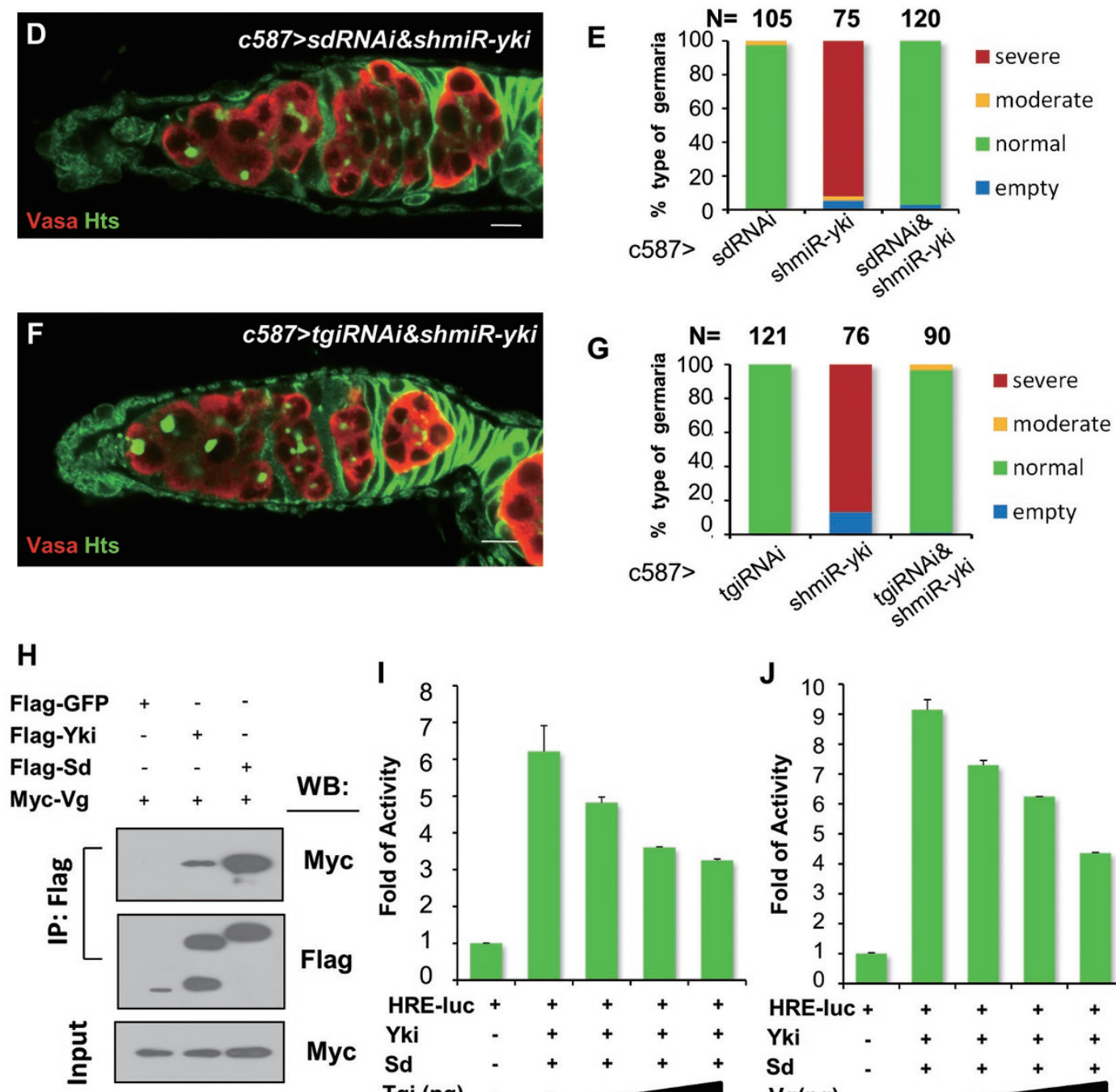

I

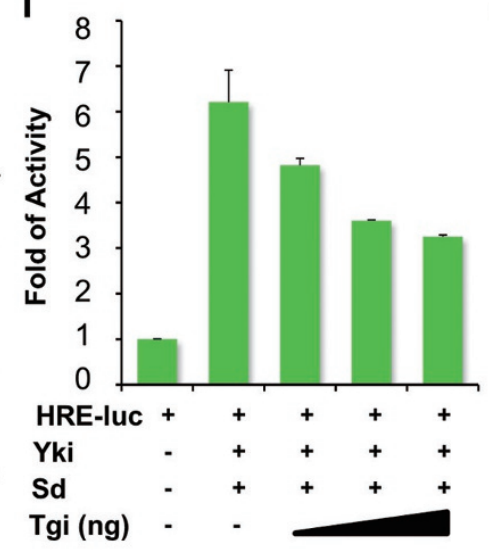

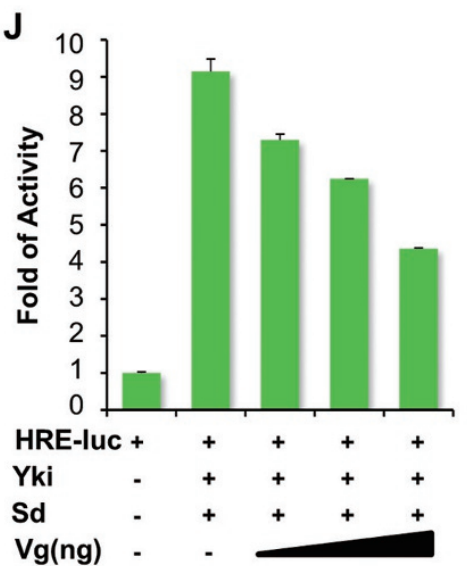

Figure 2 Yki maintains EC function through the canonical Hippo pathway. (A-C) Examples of three types of germarium phenotypes, including normal-like germaria (number of Spectrosomes (Sp): 2-7) (A), moderate tumorous germaria (number of Sp: 8-20) (B) and severe tumorous germaria (number of Sp: > 20) (C). (D-G) Ovaries from c587; $P\{$ uas $P$-shmiR-yki\}/P $\{$ uassdRNAi\} (D) and c587; $\mathrm{P}\{$ uasP-shmiR-yki\}/P\{uas-tgiRNAi\} (F) females were stained with anti-Hts (green) and anti-Vasa (red) antibodies. Histogram of corresponding phenotypes is shown in $\mathbf{E}$ and $\mathbf{G}$, respectively. Scale bar, $10 \mu \mathrm{m}$. (H) S2 cells were transfected with different combinations of DNA constructs as indicated. Forty-eight hours post transfection, cell lysates were immunoprecipitated with anti-Flag M2 affinity gel. Western blot was performed to detect the presence of the Myc-tagged or Flag-tagged proteins. Input was detected by anti-Myc antibody. (I-J) S2 cells were transfected with Flag-Yki (100 ng) and Flag-Sd (100 ng) with reporter constructs, HRE-Luc (60 ng) and renilla (40 ng). Of note, Flag-Tgi (I) or Myc-Vg (J) was transfected into cells in a gradient dosage (50 ng, $100 \mathrm{ng}$, and $200 \mathrm{ng}$ ). The experiments were carried out in triplicates, and error bars represent standard deviations calculated by Excel. 
(Supplementary information, Figure S2F). However, like Sd, overexpression of Tgi failed to induce tumorous germarium phenotype (Supplementary information, Figure S2G). These findings suggest that an unknown $\mathrm{Sd}$ cofactor in the default repression complex plays the instructive role in controlling the function of ECs to support germline development. A couple of clues drove our attention on another Tondu-domain-containing protein, $\mathrm{Vg}$, in Drosophila. First, Vg could form a complex with $\mathrm{Sd}$ in S2 cells (Figure 2H) [21, 22]. Here, we want to note that although they are male cells $[34,35], \mathrm{S} 2$ cells have been widely used in Drosophila biochemical studies. Cell culture system sometimes may not recapitulate what is happening in vivo; however, results from cultured cells could provide biochemical hints for mechanistic studies. Second, as shown in cell-based reporter assays, overexpression of $\mathrm{Vg}$ significantly antagonized Yki activity, resembling the effect of Tgi overexpression (Figure 2I-2J). These observations prompted us to investigate whether Vg acts as the instructive factor in the default repression complex in ECs. As shown in Figure 3A-3B and Supplementary information, Figure S3A-S3B', an apparent tumorous germanium phenotype was produced when we initiated $\mathrm{Vg}$ overexpression after animal eclosion by manipulating the temperature using the method described above. Notably, vg knockdown evidently suppressed the tumorous germarium phenotype induced by $y k i$ knockdown, although knockdown of $v g$ alone driven by $c 587$ gal4 exhibited no apparent germarium phenotype (Figure 3C-3D and Supplementary information, Figure S3C). Conversely, the tumorous germarium phenotype induced by overexpression of $\mathrm{Vg}$ was significantly suppressed by simultaneous expression of Yki in ECs (Figure 3E-3F). Thus, our findings indicate that $\mathrm{Vg}$ likely plays an instructive role in mediating the default repression in ECs.

To better understand the biochemical nature of the default repression complex, we performed protein competition assays. As shown in Figure 3G-3H, like Tgi, overxpression of $\mathrm{Vg}$ disrupted the interaction of Yki with $\mathrm{Sd}$ in a dose-dependent manner. We noted that, while it indeed impaired the interaction of Tgi with Sd (Figure 3I) [24], overexpression of Yki failed to disrupt the $\mathrm{Vg}$-Sd interaction under our experimental conditions (Supplementary information, Figure S3D), suggesting that Yki primarily competes with Tgi to bind Sd. We next performed further genetic tests, and found that EC-specific tgi knockdown suppressed the tumorous germarium phenotype induced by overexpression of $\mathrm{Vg}$ in ECs (Figure 3J-3K). Collectively, our findings suggest that the proper differentiation of early germ cells is maintained by Yki, which suppresses Sd-Tgi-Vg-mediated default repression pathway in ECs.
Hh signaling supports niche function by modulating Hippo signaling

Having shown that Yki regulates EC niche function via the canonical Hippo pathway by antagonizing Sd-Tgi-Vg-mediated default repression, we next sought to explore the mechanism by which Yki activity is regulated in ECs. Given that activation of the Hippo kinase cascade causes inactivation of Yki $[19,36]$ in the canonical Hippo pathway, we speculated that overexpression of Hpo or Wts in ECs would produce similar tumorous germarium phenotype as seen in $y k i$-knockdown ovaries. Unexpectedly, however, we found that overexpression of Hpo or Wts alone, or even overexpression of Hpo and Wts in combination in ECs produced normal oogenesis (Supplementary information, Figure S1D-S1E and S1P), indicating the existence of an uncharacterized mechanism in ECs that supports Yki activation by limiting the activity of the Hpo-Wts kinase cascade.

In line with its function, Yki is predominantly expressed in somatic cells in germaria, including ECs [37] (Supplementary information, Figure S1A-S1A). This is similar to the expression pattern of Ci (Supplementary information, Figure S4A-S4A") [38], the key transcriptional effector in the $\mathrm{Hh}$ signaling pathway. We therefore explored whether the Hh pathway is involved in the regulation of Yki signaling in ECs and thus maintains the proper development of early germline. The Hh pathway plays evolutionarily conserved roles in controlling a wide variety of developmental processes, such as pattern formation, growth control and stem cell regulation [39, 40]. In Drosophila ovary, loss of $h \mathrm{~h}$ results in follicle stem cell loss and defects in germ cell differentiation [28, 38, 41]. Furthermore, $\mathrm{Hh}$ is highly expressed in $\mathrm{CpCs}$ and TF cells, but is present in ECs only at very low levels (Supplementary information, Figure S4B) [38]. In contrast, the Hh-responsive target Ptc is predominantly present in ECs (Supplementary information, Figure S4C). To explore whether Hh pathway and Yki act in a common pathway to regulate germ cell differentiation, we first examined $h h$-mutant ovaries from 7-day-old $h h^{A C 2} / h h^{T_{S}}$ females, which had been shifted to the non-permissive temperature $\left(29^{\circ} \mathrm{C}\right)$ after eclosion. Consistent with previous findings, loss of $h h$ led to a defect in germ cell differentiation, and a significant number of GSC/CB-like cells were ectopically induced in a portion $(\sim 60 \%, n=126)$ of hh-mutant germaria (Figure 4A and 4C), suggesting that $\mathrm{Hh}$ signaling contributes to the proper differentiation of early germ cells. Hh is not only emitted from $\mathrm{CpCs}$, but also is expressed at low levels in ECs. Based on this, we performed additional genetic experiments. Although knockdown of $h h$ alone either in CpCs (by bab-gal4) or in ECs (by c587-gal4) causes no apparent germar- 

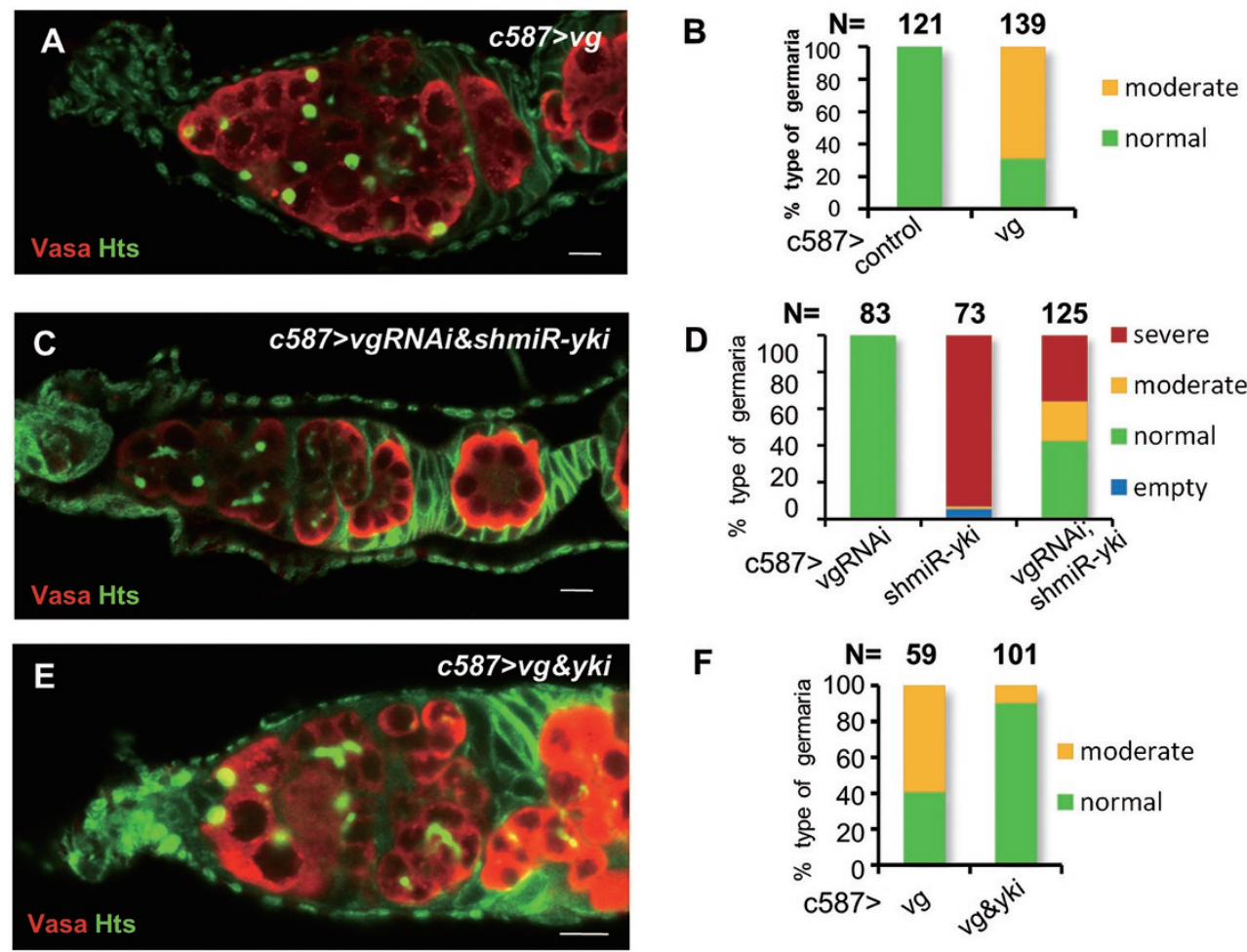

$\mathbf{F}$
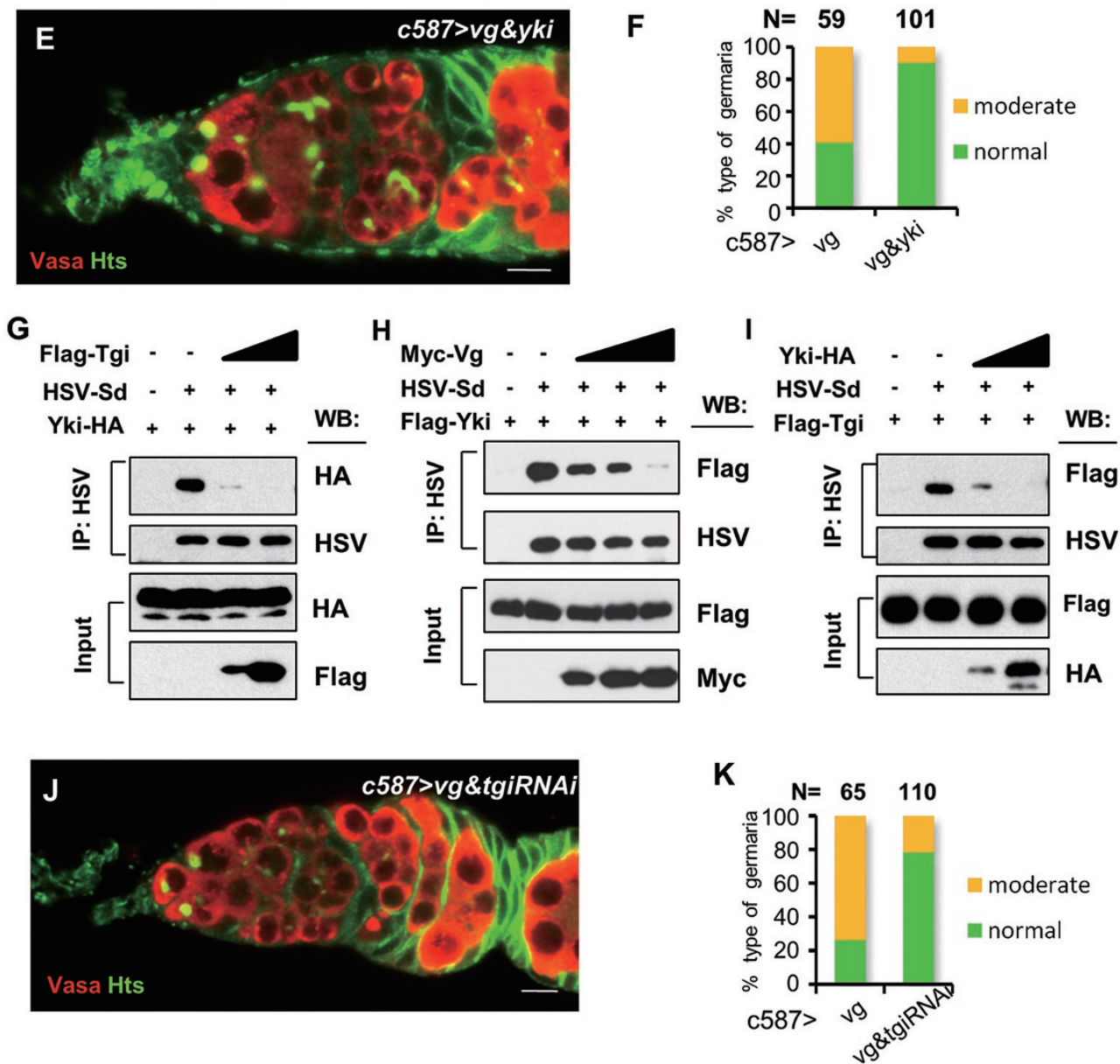

Figure $3 \mathrm{Vg}$ plays an instructive role in Sd-mediated default repression in ECs. (A-B) Ovaries from c587; $\mathrm{P}\{u a s-v g\}$ were stained with anti-Hts (green) and anti-Vasa (red) antibodies (A). Statistics of corresponding phenotypes is shown in B. (C-D) Ovaries from c587; $\mathrm{P}\{$ uasP-shmiR-yki\}/P\{uas-vgRNAl\} were stained with anti-Hts (green) and anti-Vasa (red) antibodies (C). Histogram of corresponding phenotypes is shown in D. (E-F) Ovaries from c587; P\{uas-vg\}/P\{uas-yki\} females were stained with anti-Hts (green) and anti-Vasa (red) antibodies (E). Histogram of corresponding phenotypes is shown in F. (G-I) S2 cells were transfected with the indicated DNA constructs, and Flag-Tgi $(\mathbf{G})$, Myc-Vg (H) and Yki-HA (I) were transfected into S2 cells in a gradient dosage. Lysates from transfected cells at $48 \mathrm{~h}$ post transfection were immunoprecipitated with the indicated antibodies, and western blot was performed to detect the presence of the indicated proteins. (J-K) Ovaries from c587; $\mathrm{P}\{$ uas-vg\}; P\{uas-tgiRNAl\} females were stained with anti-Hts (green) and anti-Vasa (red) antibodies (J). Histogram of corresponding phenotypes is shown in K. Scale bar, $10 \mu \mathrm{m}$. 
ium phenotype (data not shown), knockdown of $h h$ in $\mathrm{CpCs}$ and $\mathrm{ECs}$ together leads to tumorous germarium phenotype (Supplementary information, Figures S4D), suggesting that $\mathrm{Hh}$ molecules produced in $\mathrm{CpCs}$ indeed contribute to the EC function and differentiation of early germ cells.

We noted that loss of $h h$ produced a moderate tumorous germarium phenotype, which was much milder than that observed in $y k i$-knockdown ovaries. To test the potential regulatory link between Hippo and Hh signaling in regulating EC niche function, we overexpressed hpo using c587-gal4 in the $h h$ mutant background, and found that overexpression of hpo significantly aggravated $h h$ mutant-induced tumorous germarium phenotype (Figure 4B-4C). This phenotype evidently mimics that observed in $y k i$-knockdown ovaries. Thus, our findings indicate that Hh signaling likely inhibits the Hippo kinase cascade activity to support Yki activation in ECs.

\section{Ci functions in ECs by blocking Hpo-Wts kinase cascade activity}

To better understand the role of $\mathrm{Hh}$ signaling in ECs, we further investigated the role of $\mathrm{Ci}$, the key effector of the Hh pathway, by performing RNAi experiments. Following our previous method [31], we generated an shmiR-ci line, which showed significantly decreased levels of the Ci protein and ptc-lacZ when shmiR-ci was expressed in wing disc cell clones (Supplementary information, Figure S4E). To study the role of $\mathrm{Ci}$ in ovaries, we specifically knocked down $c i$ in germ cells and ECs using nosP-gal4:vp 16 and c587-gal4, respectively. As shown in Supplementary information, Figure S4F, knockdown of $c i$ driven by nosP-gal4:vp16 induced no apparent phenotype in ovaries. By contrast, knockdown of $c i$ driven by $c 587$-gal4 resulted in moderate tumorous germarium phenotypes, although these phenotypes were apparently milder than those observed in $y k i$-knockdown ovaries (Figure 4D and 4F). To avoid off-target effects and gain more evidence to confirm our conclusion, we used another ciRNAi line and an smoRNAi line, and obtained similar results (Supplementary information, Figure S4G-S4H). Interestingly, we found that the ectopic GSC/CB-like cells induced by EC-specific $c i$ knockdown expressed bam-GFP but not pMad (Supplementary information, Figure S4I-S4J) in majority of germaria (if not all), suggesting that in ECs, Ci likely acts in parallel with Dpp signaling to promote early germ cell differentiation. To establish the link between $\mathrm{Ci}$ and $\mathrm{Hpo}$, we overexpressed hpo in both wild-type and $c i$-knockdown backgrounds using the c587-gal4 driver. As shown in Supplementary information, Figure S1D and Figure 4D$4 \mathrm{~F}$, overexpression of Hpo alone in the wild-type back- ground led to no apparent germarium phenotype, whereas overexpression of Hpo in the $c i$-knockdown background produced a strong tumorous germarium phenotype, which mimics the phenotypes that we observed in $y k i$-knockdown ovaries. Similar results were consistently obtained when wts was overexpressed in $c i$-knockdown ovaries (Supplementary information, Figure S4K). Thus, our results indicate that $\mathrm{Ci}$ maintains the function of ECs likely by antagonizing Hpo-mediated kinase cascade activity. To further test this idea, we examined whether reduction of hpo expression could alleviate germarium phenotype induced by $c i$ knockdown. As shown in Figure 4G-4I, knockdown of hpo significantly suppressed ci knockdown-induced tumorous germarium phenotype. To obtain additional evidence to support our argument, we blocked Hippo signaling by knockdown of wts in transgenic lines carrying both $c i$ knockdown and Hpo overexpression in ECs, and found that wts knockdown apparently suppressed tumorous germarium phenotype induced by overexpression of Hpo in $c i$-knockdown ovaries (Figure 4J-4L). Taken together, our findings support that one important function of $\mathrm{Ci}$ in $\mathrm{ECs}$ is to provide a barrier against the Hippo kinase cascade activity.

\section{Ci maintains effective Yki activity to antagonize Sd-medi- ated default repression}

On the basis of above observations, we reasoned that $\mathrm{Ci}$ activity might contribute to maintaining effective Yki activity and thus antagonizing Sd-mediated default repression in ECs. To test this hypothesis, we determined the relationship between $\mathrm{Ci}$ and $\mathrm{Yki}$ in vivo by altering their expression levels in the germarium. As shown in Supplementary information, Figure S5A-S5D, overexpression of the activated form of $\mathrm{Ci}\left(\mathrm{Ci}^{\mathrm{CA}}\right)$ failed to suppress the tumorous germarium phenotype induced by knockdown of $y k i$ in ECs. In contrast, overexpression of Yki not only reversed, albeit not fully, the moderate tumorous germarium phenotype induced by knockdown of $c i$ in ECs, but also siginificantly suppressed the strong tumorous germarium phenotype induced by overexpression of Hpo in $c i$-knockdown ovaries (Figure 5A-5D). These findings suggest that $\mathrm{Ci}$ acts upstream of Yki in ECs. To gain more evidence, we performed further genetic assays to determine whether $\mathrm{Ci}$ contributes to Sd-mediated default repression. As shown in Figure 5E-5J, knockdown of either $s d$, tgi or $v g$ driven by $c 587$-gal4 evidently suppressed the tumorous germarium phenotype induced by $c i$ knockdown. These findings together emphasize a linear sequential relationship between $\mathrm{Ci}, \mathrm{Hpo} / \mathrm{Wts}$, Yki and Sd-mediated repression complex.

\section{Ci influences the Hippo signaling via interaction with}



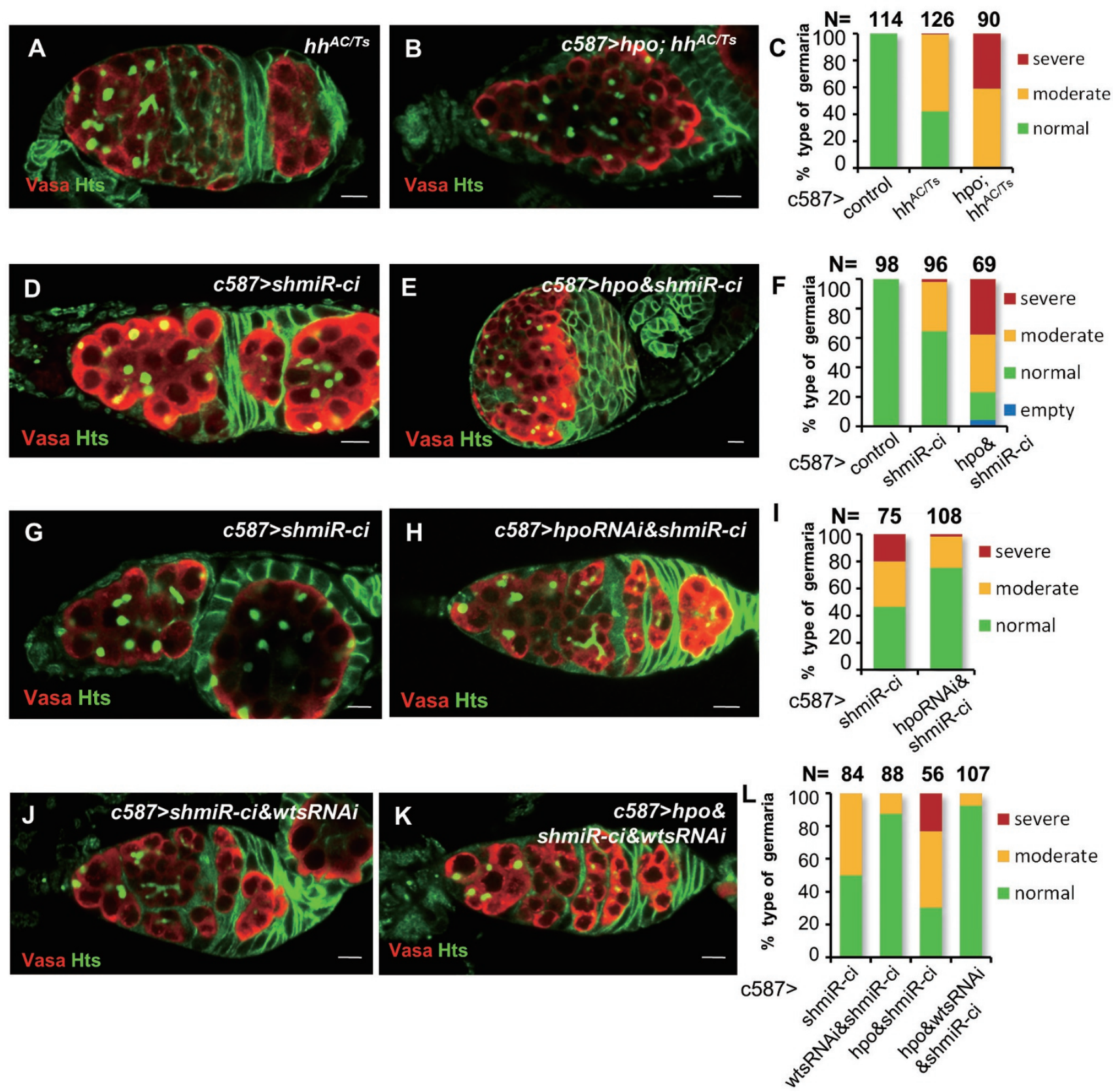

Figure $4 \mathrm{Ci}$ functions in ECs by blocking Hpo-Wts kinase cascade activity. (A-C) Ovaries from $h h^{A C 2} / h h^{T s}$ (A), and $c 587$; $\mathrm{P}\{$ uas- $h p o\} ; h h^{A C 2} / h h^{T s}(\mathrm{~B})$ females were stained with anti-Hts (green) and anti-Vasa (red) antibodies. Histogram of corresponding phenotypes is shown in C. (D-F) Ovaries from c587; $\mathrm{P}\{$ uasP-shmiR-cl\} (D) and c587; $\mathrm{P}\{$ uas-hpo\}; $\mathrm{P}\{$ uasP-shmiR$\mathrm{Cl}\}$ (E) females were stained with anti-Hts (green) and anti-Vasa (red) antibodies. Histogram of corresponding phenotypes is shown in F. (G-L) Ovaries from c587; $\mathrm{P}\{$ uasP-shmiR-cl\} (G), c587; $\mathrm{P}\{$ uas-hpoRNAi\}; $\mathrm{P}\{$ uasP-shmiR-ci\} (H), c587; $\mathrm{P}\{$ uasPshmiR-cl\}/P $\{$ uas-wtsRNAl\} (J), and c587; $\mathrm{P}\{$ uas-hpo\}; $\mathrm{P}\{$ uas P-shmiR-cl\}/P $\{$ uas-wtsRNAl\} (K) were stained with anti-Hts (green) and anti-Vasa (red) antibodies. Histogram of corresponding phenotypes is shown in $\mathbf{I}$ and $\mathbf{L}$.

\section{Hpo and $Y k i$}

We next sought to elucidate the molecular mechanism underlying the biochemical action of $\mathrm{Ci}$ in regulating the Hippo signaling. Given the antagonistic effect of $\mathrm{Ci}$ on Hpo-mediated kinase cascade, we first tested whether $\mathrm{Ci}$ is associated with core components of the Hippo kinase cascade by performing co-immunoprecipitation (co-IP) experiments in S2 cultured cells. As shown in Figure 6A and Supplementary information, Figure S6A-S6C, while Wts, Sav and Mats failed to be detected in the Ci immu- 
noprecipitates, Hpo was found to be strongly associated with $\mathrm{Ci}$, suggesting that $\mathrm{Ci}$ is selectively associated with Hpo, but not Wts, Sav or Mats. These findings raised an intriguing question of whether $\mathrm{Ci}$ influences Hippo signaling complex assembly by targeting Hpo. The core kinase cascade of the Hippo pathway comprises Hpo and Wts kinases, and their scaffold proteins, Sav and Mats. The Hpo-Wts complex could be easily detected in the presence of the Sav scaffold protein (Figure 6B). Interestingly, overexpression of $\mathrm{Ci}$ appeared to impair the formation of the Hpo-Wts complex (Figure 6B), but not that of the Hpo-Sav complex (Supplementary information, Figure S6D). Taken together, our findings support that $\mathrm{Ci}$ antagonizes the Hippo kinase complex activity via targeting the Hpo protein.

To test whether the role of $\mathrm{Ci}$ in regulating Hippo kinase complex activity contributes to Yki signaling activation via regulating Yki phosphorylation, we measured phosphorylation levels of Yki upon ectopic expression of $\mathrm{Ci}$ in $\mathrm{S} 2$ cells, and found that expression of $\mathrm{Ci}$ reduced levels of phosphorylation of Yki mediated by Hpo or Wts (Figure 6C-6D and Supplementary information, Figure S6E-S6F). Additionally, we found that the level of Wts phosphorylation mediated by Hpo was reduced when $\mathrm{Ci}$ was overexpressed (Supplementary information, Figure S6G).

Next, we investigated whether Ci directly affects Yki function and performed co-IP experiments. As shown in Figure $6 \mathrm{E}$ and Supplementary information, Figure S6HS6I, Ci was detected to be associated with Yki, but not $\mathrm{Sd}$ or Tgi, in S2 cells. Consistent with these observations, transfected $\mathrm{Ci}$ was also detected to form a complex with endogenous Yki in S2 cells (Figure 6F). Since Ci and Yki are present in both cytosol and nuclear compartments, we therefore performed fractionation experiments followed by co-IP assays. As shown in Supplementary information, Figure S6J, the Ci-Yki association was detected in both nuclear and cytosolic fractions. To define the specific region of $\mathrm{Ci}$ that is required for the $\mathrm{Ci}-\mathrm{Hpo}$ and $\mathrm{Ci}$-Yki associations, we performed domain-mapping experiments. As shown in Supplementary information, Figure S6K-S6L, Ci-155 and C-terminal half form of Ci (Ci75-155), but not its truncated form Ci-75 (N-terminal half form of $\mathrm{Ci}$ ), could associate with Yki, whereas Ci75 exhibited stronger association with Hpo compared with Ci75-155, suggesting that $\mathrm{Ci}$ associates with Hpo and Yki via its distinct molecular domains. Given that the Hippo kinase cascade blocks nuclear translocation of $\mathrm{Yki}$, and that $\mathrm{Ci}$ interacts with Yki, we asked whether overexpression of $\mathrm{Ci}$ affects the sub-cellular localization of Yki. The fractionation experiment revealed that expression of $\mathrm{Ci}$ evidently promoted accumulation of $\mathrm{Yki}$ in the nuclear fraction in $\mathrm{S} 2$ cells (Figure 6G-6H). The immunostaining assay further confirmed that expression of $\mathrm{Ci}$ increased Yki nuclear translocation (Figure 6I-6J). Thus, our biochemical experiments suggest that $\mathrm{Ci}$ influences formation of the Hpo/Wts signaling complex and ensures effective Yki signaling by promoting its nuclear translocation.

Mathematical modeling: bi-stable behavior of Yki signaling determined by the antagonistic effect of $\mathrm{Ci}$ on the Hpo kinase cascade

Our experimental data strongly suggest that the antagonism of Hippo signaling by $\mathrm{Ci}$ determines the effective Yki activity to maintain the EC niche function that promotes early germ cell differentiation. This process can be briefly summarized as: (i) the Hpo/Wts kinase cascade catalyzes the phosphorlation of Yki that leads to the inactivation of $\mathrm{Yki}$ (Yki $\stackrel{\mathrm{Hpo} / \mathrm{Wts}}{\longrightarrow} \mathrm{Yki}^{\mathrm{P}}$ ); (ii) Ci can not only inhibit the activity of $\mathrm{Hpo} / \mathrm{Wts}(\mathrm{Hpo} / \mathrm{Wts} \stackrel{\mathrm{Ci}}{\longrightarrow}$ inactivity) but also bind Yki to form the complex Ci-Yki (Ci-Yki denotes the complex of $\mathrm{Ci}$ and $\mathrm{Yki}$ ), and the disintegration of Ci-Yki will release Yki $(\mathrm{Ci}+\mathrm{Yki} \underset{\lambda 2}{\stackrel{\lambda 1}{\rightleftarrows}} \mathrm{Ci}-\mathrm{Yki})$; and (iii) the activity of $\mathrm{Yki}$ is promoted by the synthesis rate of itself in a positive feedback manner (Supplementary information, Figure S6M-6N). The diagrammatic sketch of this system is plotted in Figure 7A. However, a more interesting and challenging question is how the dynamic properties of Yki are determined by the antagonistic effect of $\mathrm{Ci}$ on $\mathrm{Hpo} / \mathrm{Wts}$, which controls the $\mathrm{EC}$ niche function that contributes to the proper differentiation of early germ cells. A theoretical model based on the experimental evidence was developed to illustrate how the bi-stable behavior of Yki signaling is induced and controlled by the antagonism of $\mathrm{Ci}$ against $\mathrm{Hpo} / \mathrm{Wts}$.

To assess the dynamic properties of the system shown in Figure 7A, the concentrations of $\mathrm{Hpo} / \mathrm{Wts}, \mathrm{Ci}$, Yki, phosphorlated Yki (Yki ${ }^{\mathrm{P}}$ ) and complex Ci-Yki are denoted by $x_{H}, x_{C}, x_{Y}, x_{Y^{P}}$ and $x_{C-Y}$, respectively. The deterministic rate equation of the system can be written as:

$$
\begin{aligned}
& \frac{d x_{H}}{d t}=f_{H}-\phi_{H}\left(x_{C}\right) x_{H}, \\
& \frac{d x_{C}}{d t}=f_{C}-\phi_{C} x_{C}-\lambda_{1} x_{C} x_{Y}+\lambda_{2} x_{C-Y}, \\
& \frac{d x_{Y}}{d t}=f_{Y}\left(x_{Y}\right)-\phi_{Y} x_{Y}-\lambda_{1} x_{C} x_{Y}+\lambda_{2} x_{C-Y}-\vartheta_{Y^{p}}\left(x_{H}\right) x_{Y}, \\
& \frac{d x_{Y^{P}}}{d t}=\vartheta_{Y^{p}}\left(x_{H}\right) x_{Y}-\phi_{Y^{p}} x_{Y^{P}}, \\
& \frac{d x_{C-Y}}{d t}=\lambda_{1} x_{C} x_{Y}-\lambda_{2} x_{C-Y},
\end{aligned}
$$

where $f_{H}, f_{C}, f_{Y}\left(x_{Y}\right), \vartheta_{Y^{P}}\left(x_{H}\right)$ are the synthesis rates of 

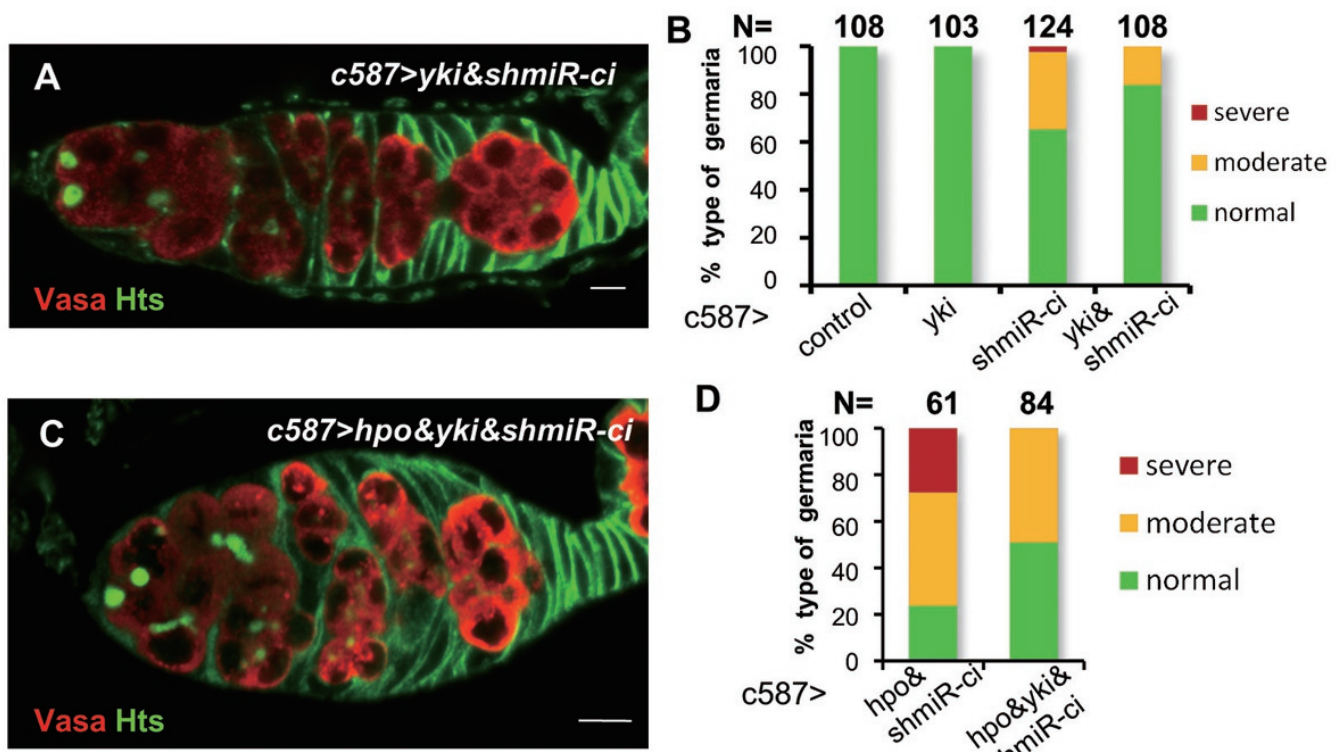

D
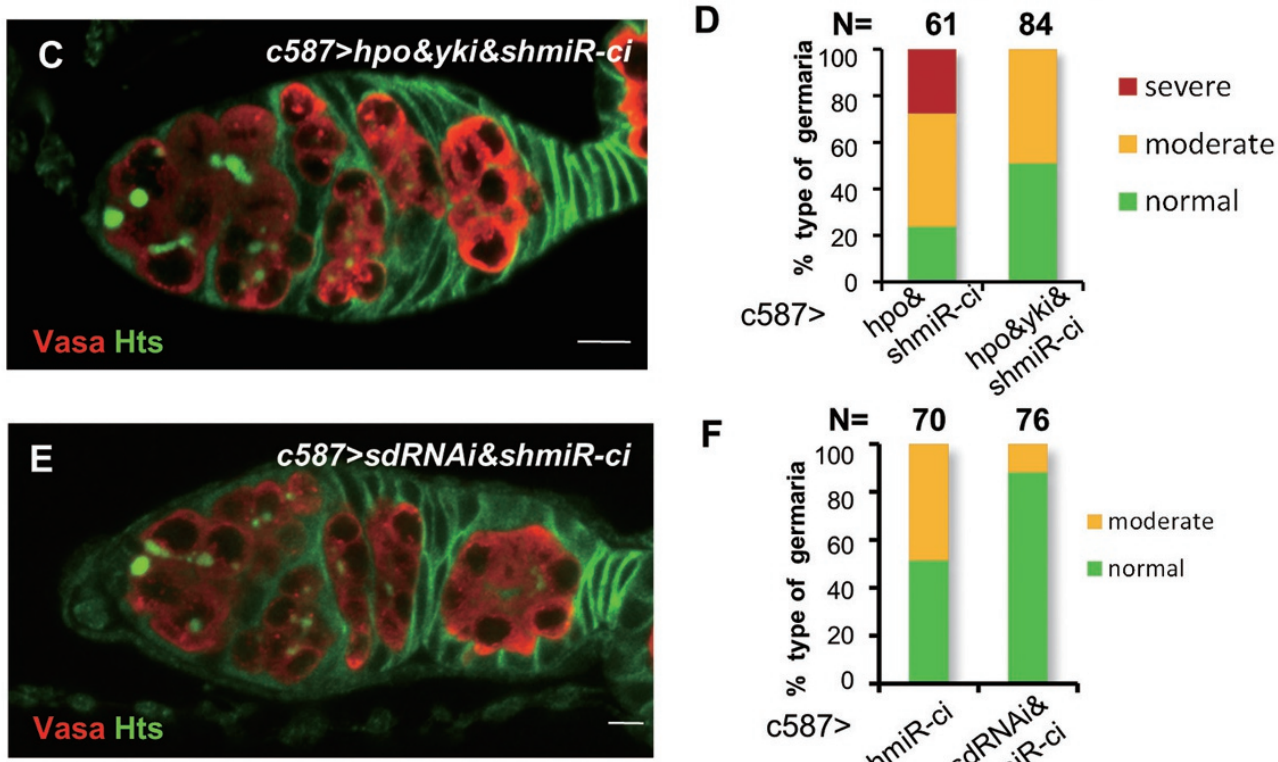

$\mathbf{F}$
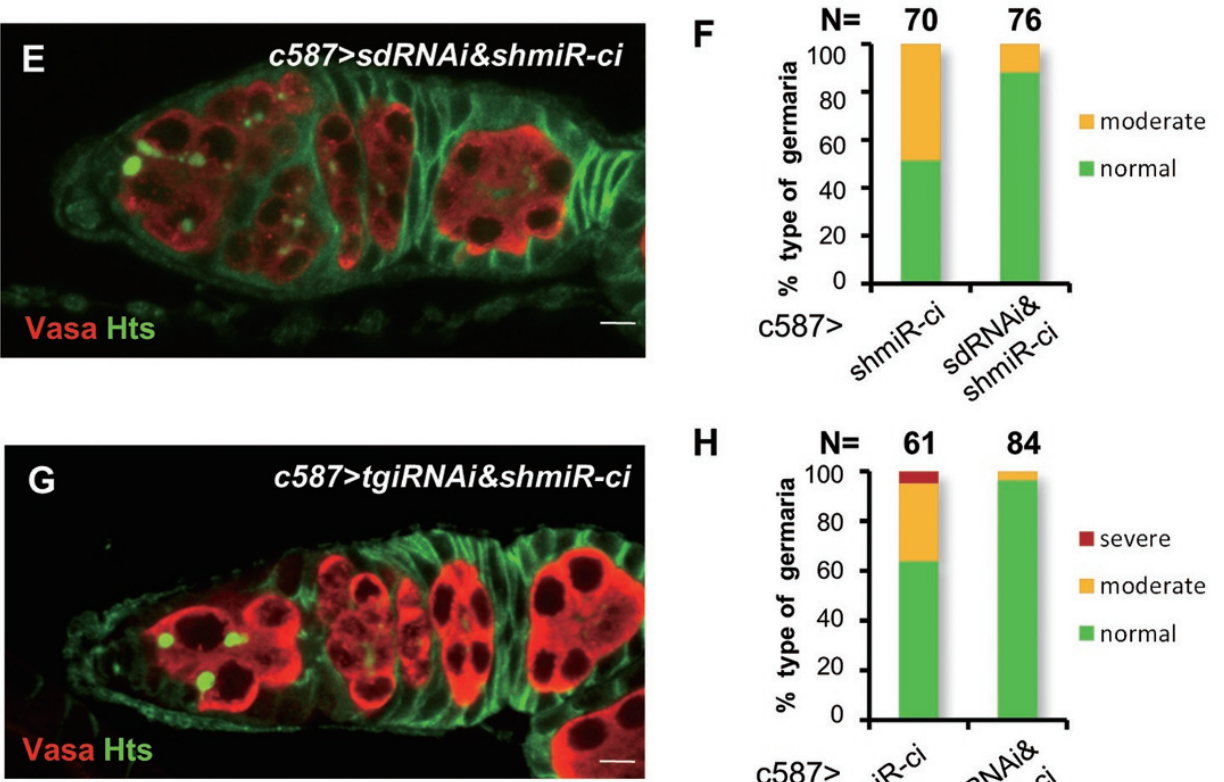

H
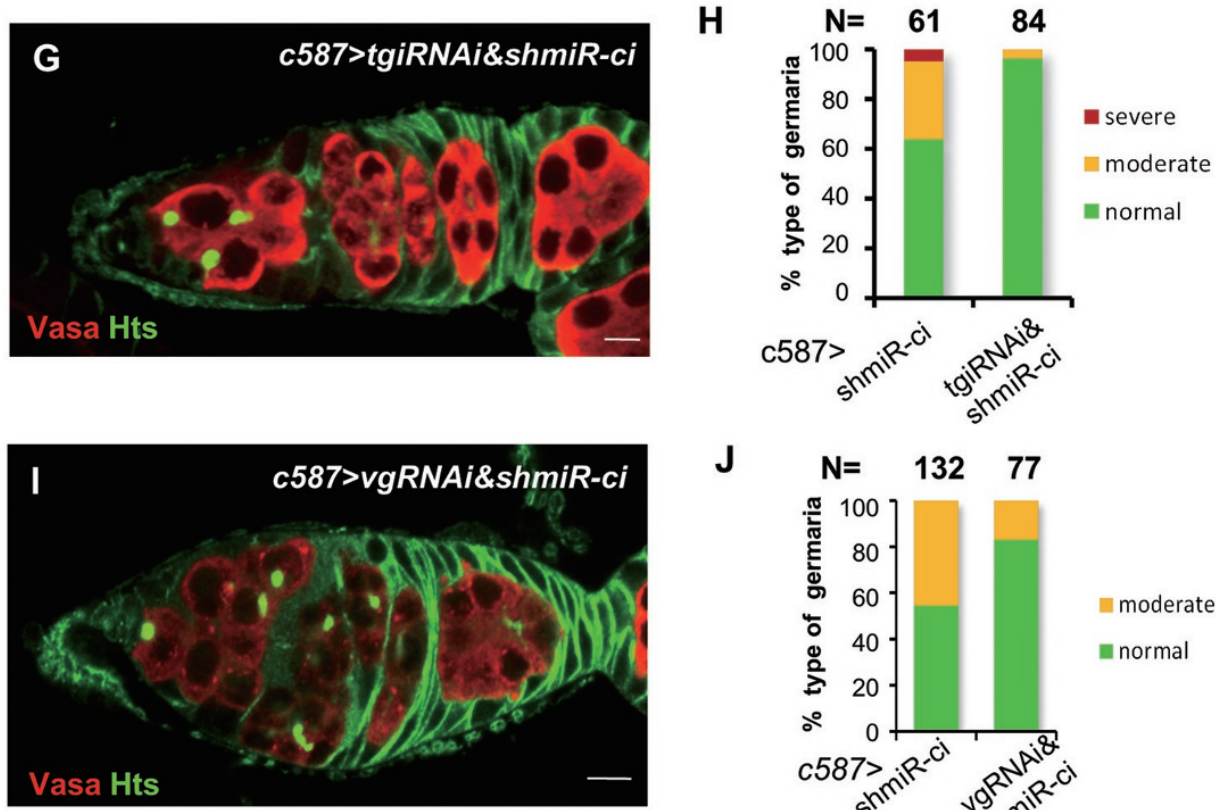

$\mathbf{J}$

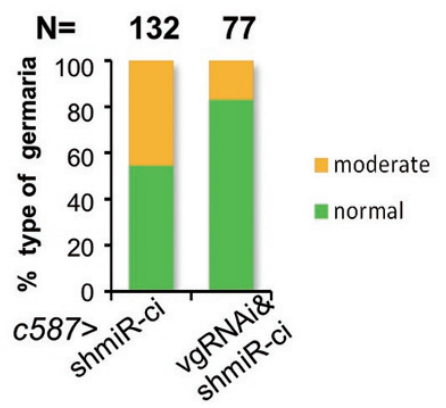

Figure $5 \mathrm{Ci}$ maintains effective Yki activity to antagonize Sd-mediated default repression. (A-J) Ovaries from c587; $P\{$ uasykl\}; $\mathrm{P}\{$ uasP-shmiR-cl\} (A), c587; $\mathrm{P}\{$ uas-hpo\}/P $\{$ uas-ykl\}; $\mathrm{P}\{$ uasP-shmiR-cl\} (C), c587; $\mathrm{P}\{$ uas-sdRNAI\}/P $\{$ uasP-shmiR-cl\} (E), c587; $\mathrm{P}\{$ uas-tgiRNAi\}/P\{uasP-shmiR-cl\} (G) and c587; $\mathrm{P}\{$ uas-vgRNAl\}; $\mathrm{P}\{$ uasP-shmiR-cl\} (I) were stained with anti-Hts (green) and anti-Vasa (red) antibodies. Histogram of phenotypes corresponding to A, C, E, G and I is shown in B, D, F, H and J, respectively. Scale bar, $10 \mu \mathrm{m}$. 
$\mathrm{Hpo} / \mathrm{Wts}, \mathrm{Ci}$, Yki and $\mathrm{Yki}^{\mathrm{P}}$, respectively, with $d f_{Y}\left(x_{Y}\right) /$ $d x_{Y}>0$ and $d \vartheta_{Y^{P}}\left(x_{H}\right) / d x_{H}>0 ; \Phi_{H}\left(x_{C}\right), \Phi_{C}, \Phi_{Y}$ and $\Phi_{Y^{P}}$ are degradation rates of $\mathrm{Hpo} / \mathrm{Wts}, \mathrm{Ci}$, Yki and $\mathrm{Yki}^{\mathrm{P}}$, respectively, with $d \Phi_{H}\left(x_{C}\right) / d x_{C}>0$; and $\lambda_{1}$ and $\lambda_{2}$ are the integration and disintegration rates of the complex $\mathrm{Ci}$ Yki, respectively. We here take the synthesis rate of Yki, $f_{Y}\left(x_{Y}\right)$, as a Hill-type function $f_{Y}\left(x_{Y}\right)=k_{\max } x_{Y}^{2} /\left(K^{2}+x_{Y}^{2}\right)+k_{0}$, where the parameter $k_{\max }$ stands for the maximum synthesis rate of Yki when the level of Yki is high enough, and $k_{0}$ represents the fundamental synthesis rate of Yki when the level of Yki is very low. The equilibrium structure of dynamics (1) (Figure 7B) and stability analysis show clearly that the antagonism of $\mathrm{Ci}$ against $\mathrm{Hpo} / \mathrm{Wts}$ signaling will be able to lead to the bi-stable behavior of Yki signaling. On the other hand, for the situation with bi-stability in dynamics (1), a simple stochastic dynamic analysis (Figure 7C-7D) provides possible explanations as to why tumor and differential cells can occur simultaneously in our experiments and raises the probability that tumor (or differential cells) occurs mainly depends on the concentrations of $\mathrm{Ci}$.

\section{Overexpression of Ci amplifies the Yki signaling activity}

The Hippo and Hh signaling pathways play important roles in development $[13,39]$. On the basis of our mathematic modeling analysis, we speculated that an increase in $\mathrm{Ci}$ activity could potentiate Yki signaling in other biological processes. To test this, we employed the model of Drosophila wing disc whose development is tightly regulated by the Hh and Hippo signaling pathways. We investigated whether $c i$ is involved in regulating Hippo signaling by using the FLP-OUT system to generate mosaic clones that expressed $\mathrm{Ci}^{\mathrm{CA}}$ or harbored the knockdown of $c i$ in wing discs. Although knockdown of $c i$ did not detectably affect the expression of Yki-responsive reporters, diap1-LacZ, and ex-LacZ (data not shown), our immunostaining assays showed that the expression of diap1-LacZ (Figure 7E-7F"') and ex-LacZ (Supplementary information, Figure S7A-S7B"') in $\mathrm{Ci}^{\mathrm{CA}}$-overexpressing regions was significantly upregulated compared with that in neighboring wild-type cells. Notably, we found that knockdown of $y k i$ markedly downregulated the enhanced expression of the Yki-responsive reporters induced by $\mathrm{Ci}^{\mathrm{CA}}$ expression (Figure 7G-7H"' and Supplementary information, Figure S7C-S7D"'). Moreover, we found that overexpression of $\mathrm{Ci}^{\mathrm{CA}}$ using AP-gal4 significantly increased the size of the wing discs, but this effect was markedly suppressed by knockdown of $y k i$ (Figure 7I-7M). In support of this, we found that expression levels of diap1, ex and cyclin E, downstream target genes of $y k i$, were evidently increased in $\mathrm{Ci}^{\mathrm{CA}}$-overexpressing discs (Supplementary information, Figure S7E). To test whether Ci could activate $y k i$ downstream targets in ECs, we performed immunostaining assays, and found that overexpression of $\mathrm{Ci}^{\mathrm{CA}}$ indeed enhanced diap1-lac $\mathrm{Z}$ expression (Supplementary information, Figure S7F-S7G). By contrast, knockdown of $c i$ decreased expression levels of diap1-lacZ in ECs of timorous germaria (Supplementary information, Figure $\mathrm{S} 7 \mathrm{H}$ ). Taken together, our findings support a model in which $\mathrm{Ci}$ facilitates $\mathrm{Yki}$ signaling.

\section{Discussion}

A stem cell niche is defined as specialized cells (or tissues) forming a microenvironment that interacts with stem cells and provides secreted factors to locally regulate stem cell fate $[1,2]$. In the Drosophila ovary, a handful of somatic cells, including TF cells, $\mathrm{CpCs}$, and ECs, form a functional niche to control GSC self-renewal and differentiation in the apical region of germaria $[2$, 3]. It has been well documented that the BMP/Dpp signaling derived from niche CpCs locally suppresses GSC differentiation by silencing bam transcription in GSCs $[7,10]$. Recent studies identified several factors as niche regulators in ECs that control GSC/CB differentiation [42-44]. However, fate determination between stem cell self-renewal and differentiation must be under strict control to ensure proper development and tissue homeostasis. Therefore, a fundamental question is how the actions executed by distinct somatic cells in a niche (e.g., the cap and ECs in Drosophila ovary) are coordinated to maintain the balance of stem cell self-renewal and differentiation. In current study, we found that, in contrast to BMP signals derived from $\mathrm{CpCs}$ that locally suppress GSC differentiation, $\mathrm{Hh}$ ligands primarily produced by $\mathrm{CpCs}$ control the function of niche ECs. The downstream Hh signaling effector Ci promotes Yki activity by antagonizing the Hippo kinase cascade, thereby facilitating normal differentiation of GSC/CBs. Our findings reveal a coordinated action of two distinct cell populations in stem cell niches, which utilizes multiple signaling pathways to balance the dynamics of stem cell self-renewal and differentiation. Recent studies revealed that mutations of several other genes, such as sex-leathal, Ataxin 2-binding protein 1, and without children, also give rise to Drosophila ovarian tumors filled with bam-expressing germ cells [44-46], which mimics the phenotypes induced by knockdown of $c i$ or $y k i$ in ECs. It would be interesting to test whether these genes act in a common pathway with $c i$ and $y k i$ to regulate EC function and early germ cell differentiation.

The Hippo signaling pathway has been recently 


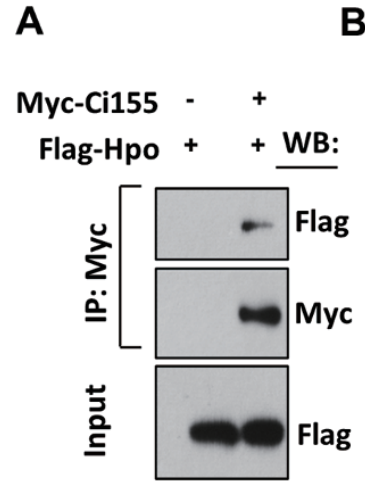

E

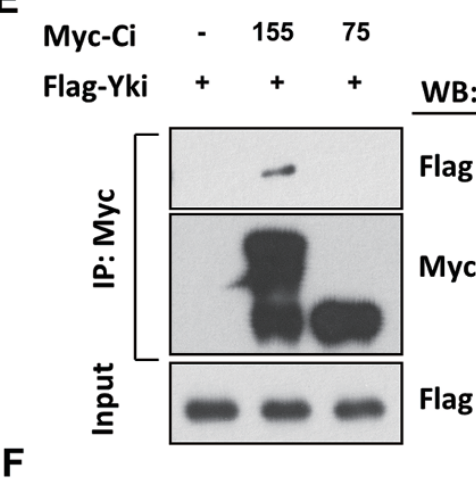

Myc-Ci155 + +

IP: IgG $\alpha$-Yki

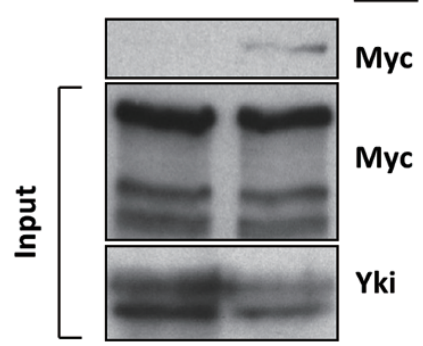

WB:

H

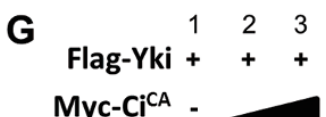
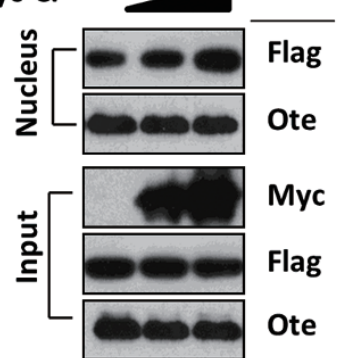

Myc

Flag

Ote

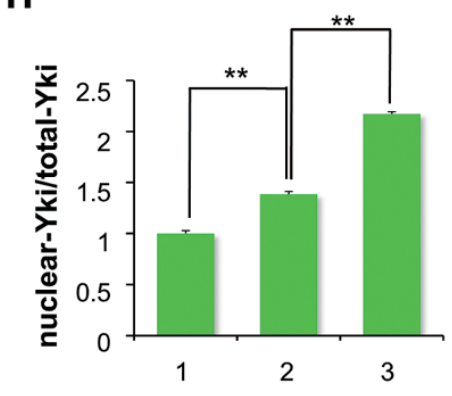

D

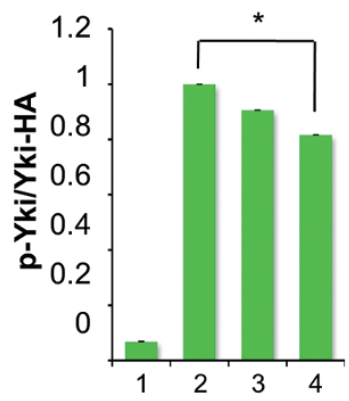

I

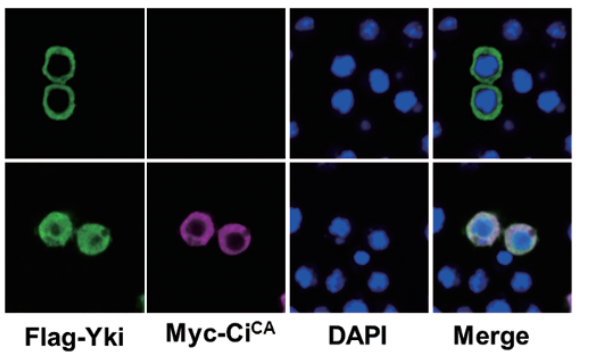

J $\quad \mathrm{N}=179200$

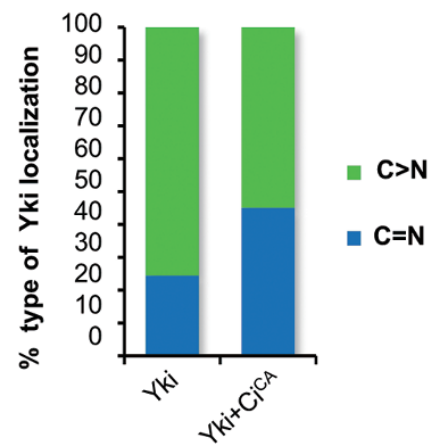

K

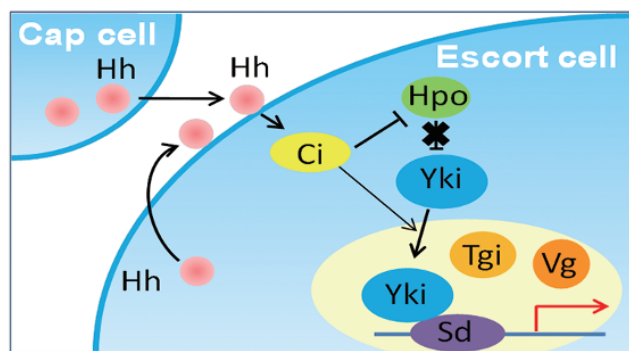

L Cap

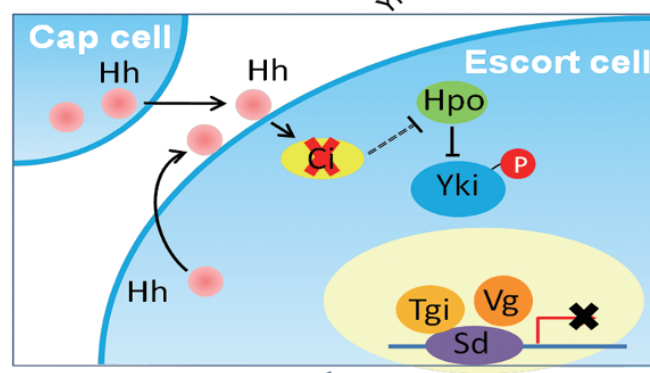

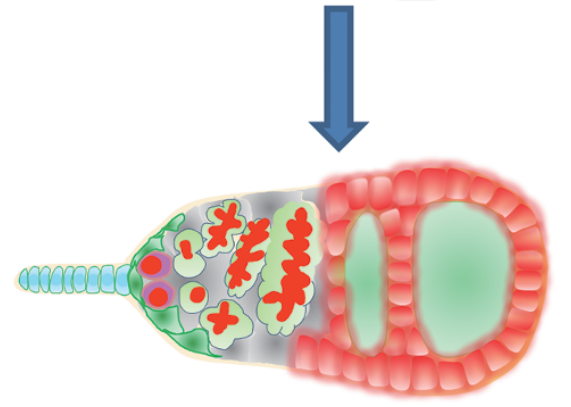

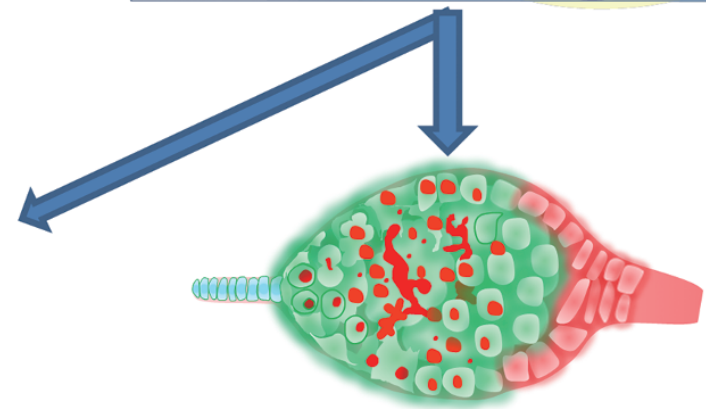

Cell Research | Vol 25 No 10 | October 2015 
Figure 6 Ci physically associates with Hpo and promotes Yki nuclear accumulation. (A) S2 cells were transfected with Flag$\mathrm{Hpo}$ and Myc-Ci155 (or empty vector). Cell lysates were immunoprecitated with anti-Myc, and western blot was performed using anti-Flag and anti-Myc. Protein input was analyzed by western blot using anti-Flag. (B) S2 cells were transfected with Flag-Hpo (or Flag-GFP), HSV-Wts and GFP-Sav in combination with different concentrations of $\mathrm{Myc}^{-\mathrm{Ci}^{\mathrm{CA}}}$, and cultured for 48 h. Flag-Hpo or Flag-GFP was immunoprecipitated and the immunoprecipitates were blotted by the indicated antibodies. (CD) S2 cells were transfected with Yki-HA and Flag-Hpo in combination with different concentrations of Myc-Ci ${ }^{\mathrm{CA}}$, and cultured for $48 \mathrm{~h}$. HA-Yki was immunoprecipitated and the immunoprecipitates were blotted by anti-pYki and anti-HA (C). D shows the relative levels of Yki phosphorylation that were quantified by the ratio of pYki to the total Yki protein levels. ${ }^{*} P<0.05$ (twotailed Student's $t$-test). (E) S2 cells were transfected with the indicated combinations of DNA constructs. Cell lysates were immunoprecipitated with anti-Flag M2 affinity gel, and western blot was performed to analyze the presence of the Myc-tagged and Flag-tagged proteins. (F) S2 cells were transfected with Myc-Ci155 (or empty vector). Cell lysates were incubated with IgG (left) or anti-Yki antibody (right), and western blot was performed using anti-Myc. (G-H) S2 cells were transfected with the indicated combinations of DNA constructs. After $48 \mathrm{~h}$, the nuclear fraction from $\mathrm{S} 2$ cell lysates was analyzed by western blot using anti-Flag and anti-Otefin. Otefin was used as a loading control for nuclear fraction. $\mathbf{H}$ shows the relative $\mathrm{Yki}$ protein levels that were quantified by the ratio of Flag-Yki to Otefin. ${ }^{* *} P<0.01$ (two-tailed Student's $t$-test). (I-J) S2 cells were transfected with Flag-Yki and Myc-Ci ${ }^{\mathrm{CA}}$ (or empty vector). Immunostaning was performed to measure the ratio of $\mathrm{Yki}$ expressed in nuclear (referred to as $\mathrm{N}$ ) to that in cytosolic compartment (referred to as $\mathrm{C}$ ). Statistic is shown in $\mathbf{J}$. (K-L) A model describing the coordinated action of $\mathrm{Hh}$ and Hippo signaling pathways that controls function of ECs and proper germline development. In this model, Hh primarily released from CpCs activates Ci in ECs. Activated Ci establishes a barrier against the Hpo kinase cascade, allowing efficient Yki signaling transduction to maintain EC function, and thereby promoting germ cell differentiation. In wild-type overy, the primary role of $\mathrm{Ci}$ is to maintain an effective Yki signaling (relative constant) in ECs to promote proper germline development, while in the absence of $\mathrm{Ci}$, Yki signaling in ECs becomes more fluctuant due to antagonistic activity of the Hpo kinase cascade. Thus, the fluctuation of Yki signaling leads to two major different phenotypes, namely, wild-type-like and tumorous germaria, as observed in our genetic assays.

demonstrated to play evolutionarily conserved roles in regulating tissue growth and organ size, as well as stem cell fate in both Drosophila and mammals [13, 47]. We questioned whether the Hippo pathway is involved in maintaining the EC function for Drosophila ovarian germline development. We performed systematic genetic tests to assess the specific roles of components in the Hippo pathway in ECs, and found that only Yki knockdown produces a defective phenotype, namely the tumorous germarium. How does Yki execute its function in ECs? Our findings clearly demonstrate that Yki regulates the niche EC cell function through the canonical Hippo pathway, because knockdown of $s d$, tgi or $v g$ is sufficient to rescue the defective phenotype induced by $y k i$ knockdown. Notably, we found that Yki does not apparently influence BMP/Dpp signaling transduction in early germ cells, although the BMP/Dpp pathway is the key protagonist in GSC maintenance. Together, our results indicate that Yki antagonizes the default repression mediated by the $\mathrm{Sd} / \mathrm{Tgi} / \mathrm{Vg}$ complex in ECs. Another open question is how the early germ cells perceive the Yki-activated signals produced in the ECs. It would be interesting to test whether other ligands from ECs induced by the Sd/Yki transcriptional complex control GSC differentiation.

We unexpectedly found that, although Yki antagonizes Sd-mediated default repression in ECs through the canonical Hippo signaling pathway, overexpression of either Hpo or Wts does not impair Yki function, suggesting the existence of an unknown mechanism in ECs that supports effective Yki signaling by blocking Hpo/Wts activation. In this study, we identified $\mathrm{Ci}$ as an Yki-supporting factor in the Drosophila germarium. A previous study proposed that $\mathrm{Hh}$ produced by $\mathrm{CpCs}$ regulates BMP expression in ECs to maintain GSCs [48], which is opposite to what we found in our study. To firmly prove our conclusion, we performed a number of assays by employing multiple strategies, including (1) using the $h h^{A C 2} / h h^{T s}$ mutants, (2) performing $h h$ knockdown in both CpCs and ECs and (3) depleting smo in ECs. Results from these experiments strongly support that $\mathrm{Hh}$ signaling in ECs promotes early germ cell differentiation. Importantly, other lines of evidence further support our model that $\mathrm{Ci}$ promotes Yki signaling by blocking Hpo/ Wts activation. First, both $\mathrm{Ci}$ and Yki are expressed in $\mathrm{ECs}$, and knockdown of $c i$ leads to the tumorous germarium phenotype, albeit much milder compared with yki knockdown. Second, while overexpression of either Hpo or Wts in ECs in a wild-type background causes no apparent germarium phenotype, expression of these Hippo components individually in the $c i$-knockdown background mimics $y k i$ knockdown-induced tumorous germarium phenotype. This suggests that $\mathrm{Ci}$ activity is the primary barrier against the inhibitory effect of the Hpo/ Wts kinase cascade on Yki. Third, knockdown of hpo or $w t s$ significantly suppresses $c i$ knockdown-induced tumorous germarium phenotype, suggesting that Hpo and Wts are targeted by $\mathrm{Ci}$ in ECs. These findings provide further evidence in support of our proposed model in 

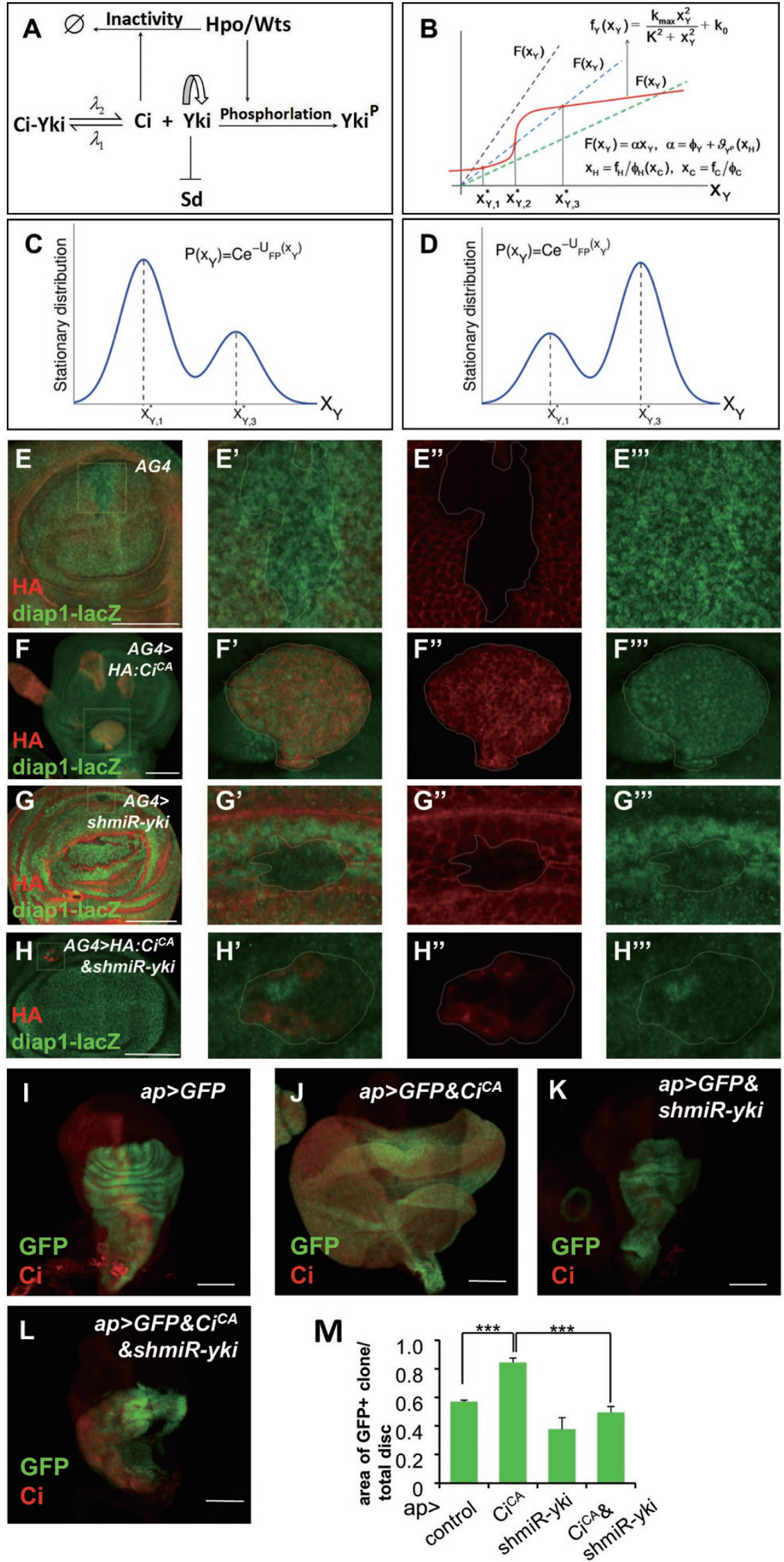
Figure 7 Overexpression of Ci amplifies the Yki signaling activity in wing discs. (A) Schematic of maintaining an effective Yki signaling via the antagonism of $\mathrm{Hpo} / \mathrm{Wts}$ by $\mathrm{Ci}$. (B) Equilibrium structure and bi-stability of dynamics (1), in which the equilibria exactly correspond to the intersects of $f_{Y}\left(x_{Y}\right)$ and $F\left(x_{Y}\right)=\alpha x_{Y}$ where $\alpha=\Phi_{Y}+\vartheta_{Y} P\left(x_{H}^{*}\right)$ since at the equilibrium $x_{H}^{*}=f_{H} / \Phi_{H}$ $\left(x^{*}\right)$ and $x_{C}^{*}=f_{C} / \Phi_{C}$. Note that $f_{Y}\left(x_{Y}\right)$ is a typical S-curve. $f_{Y}\left(x_{Y}\right)$ and $F\left(x_{Y}\right)$ have only one intersect being at high (low) level of $x_{Y}$ if the level of $x_{C}^{*}$ is high (low) (corresponding respectively to the green and purple dash lines). This implies that there should be two critical values of $x_{C}$, denoted by $x_{C}^{\prime}$ and $x{ }_{C}^{\prime}$, with $x_{C}^{\prime}<x{ }_{C}^{\prime}$, such that if $x_{C}^{*}=f_{C} / \Phi_{C}$ is in the interval $x_{C}^{\prime}<x_{C}^{*}<x_{C}{ }_{C}, f_{Y}\left(x_{Y}\right)$ and

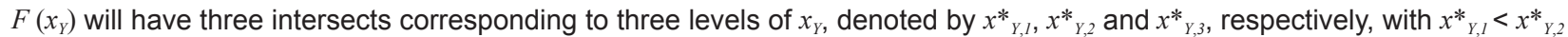
$<x_{Y, 3}^{*}$ (corresponding to the blue dash line). For the situation with three equilibrium points, the two equilibrium points corresponding respectively to $x_{Y, 1}^{*}$ and $x_{Y, 3}^{*}$ must be locally asymptotically stable, and the other corresponding to $x_{Y, 2}^{*}$ must be unstable. (C-D) For the situation with bi-stability, the stationary distribution of Yki's concentration, $P\left(x_{Y}\right)$, is a bimodal distribution with two peaks corresponding respectively to $x_{Y, 1}^{*}$ and $x_{Y, 3}^{*}$, in which the probability that the concentration of Yki is near $x_{Y, 1}^{*}$ is larger (less) than the probability that the concentration of Yki is near $x_{Y, 3}^{*}$ when the concentration of Ci is low (high). (E-H'”) In $\mathrm{P}\{$ diap1P-lacZ $\}$ background, clones of control (E), the expression of transgenes $\mathrm{P}\left\{\right.$ uas-Ci $\left.{ }^{\mathrm{CA}}: H A\right\}(\mathrm{F}), \mathrm{P}\{u a s P-s h m i R-y k i\}(\mathbf{G})$, and $\mathrm{P}\left\{\right.$ uas- $\left.\mathrm{Ci} i^{C A}: \mathrm{HA}\right\} ; \mathrm{P}\{$ uasP-shmiR-ykl\} $(\mathbf{H})$ was induced by heat shock treatment (see Supplementary information, Data $\mathrm{S} 1$ for clone induction method). Regions marked with the white rectangle in $\mathbf{E}-\mathrm{H}$ were enlarged and shown in $\mathbf{E}^{\prime}-\mathrm{H}^{\prime \prime}$. The third instar larva were stained with anti- $\beta$-gal (E'"-H'", green) and anti-HA antibody (F" and H", red) or anti-CD2 antibody (E"' and G", red). Two-color-merged images are shown in E'-H'. Scale bar, $100 \mu \mathrm{m}$. (I-M) Wing discs from P\{ap-gal4\}, P\{uas-GFP (I), $\mathrm{P}\left\{\right.$ uas-Ci $\left.{ }^{C A}: H A\right\} ; \mathrm{P}\left\{\right.$ ap-gal4\}, $\mathrm{P}\{$ uas-GFP $\}(\mathrm{J}), \mathrm{P}\left\{\right.$ uasP-shmiR-yki\}; $\mathrm{P}\left\{\right.$ ap-gal4\}, $\mathrm{P}\{$ uas-GFP $\}(\mathrm{K})$, and $\mathrm{P}\left\{\right.$ uas-Ci $\left.{ }^{\mathrm{CA}}: \mathrm{HA}\right\} ; \mathrm{P}\{$ uas $P$ shmiR-ykı\}; P\{ap-gal4\}, P\{uas-GFP\} (L) third instar larva were stained with anti-Ci antibody (red) and anti-GFP antibody (green). Clones were marked by GFP-positive cells. Scale bar, $100 \mu \mathrm{m}$. (M) Quantification of the size of the AP>GFP expression domains normalized to the size of the entire imaginal disc of the indicated genotypes. ${ }^{* \star *} P<0.001$ (two-tailed Student's $t$-test).

which $\mathrm{Ci}$ antagonizes $\mathrm{Hpo} / \mathrm{Wts}$ activation. In agreement with this, knockdown of $s d$, tgi, or $v g$, or overexpression of Yki, suppresses $c i$ knockdown-induced tumorous germarium phenotype, revealing a sequential regulatory relationship among ci, hpo/wts, yki, and sd/tgi/vg in ECs. Besides the genetic analyses, our biochemical assays suggest that $\mathrm{Ci}$ physically associates with Hpo and Yki, probably through distinct molecular domains. Importantly, our results also show that $\mathrm{Ci}$ can promote Yki nuclear distribution in a dose-dependent manner in S2 cultured cells. Thus, Ci not only functions as transcription factor to activate $\mathrm{Hh}$-responsive target genes, but also inhibits Hpo-Wts kinase cascade activity, thus enhancing Yki signaling in certain biological contexts.

The Hh and Hippo signaling pathways have been recently shown to control common biological events; however, the molecular mechanisms underlying the crosstalk between these two pathways are largely unknown [49, 50]. It was reported that the Hh signaling transcriptionally activated $y k i$ in follicle stem cells [50]. However, we found that $\mathrm{Ci}$ does not affect $y k i$ expression in ECs (Supplementary information, Figure S8), suggesting that the Hh and Hippo signaling could be regulated through different mechanisms in a context-dependent manner. Our study shows that Hh and Hippo signaling pathways are both involved in maintaining EC function and in supporting early germ cell differentiation. However, loss of $\mathrm{Ci}$ function gives rise to a much milder tumorous germarium phenotype compared with loss of Yki in ECs. Particularly, in $c i$-knockdown ovaries, although some germaria were tumorous, the majority showed normal germline development. By contrast, most $y k i$-knockdown germaria were tumorous. Although our results have integrated $\mathrm{Ci}$ into the regulatory network of the canonical Hippo signaling pathway, the biological importance of $\mathrm{Ci}$ in regulating the dynamics of this system remains unclear. How does the antagonistic effect of $\mathrm{Ci}$ on Hpo activity contribute to Yki signal transduction in ECs? Previous studies have suggested that only a low level of nuclear Yki is sufficient to maintain normal tissue growth by antagonizing Sd-mediated default repression $[24,25]$. However, this amount of nuclear Yki could be very sensitive to Hpo activation when cells respond to environmental cues during development. Our mathematic modeling analyses reveal that a bi-stable system of Yki signaling regulation could be generated in ECs when $\mathrm{Ci}$ is integrated into the system. Due to the sensitivity of Yki to the Hippo kinase cascade, in the absence of $\mathrm{Ci}$, the level of Yki in the nucleus is fluctuated. Thus, the animal produces two germarium phenotypes (tumorous and normal) in the ovaries (Figure 6K-6L). Conversely, the presence of $\mathrm{Ci}$ precludes Hpo activation and results in a high level of nuclear Yki, producing a sustained antagonism against $\mathrm{Sd}$-mediated default repression, thereby maintaining normal early germ cell development. Interestingly, a similar regulatory mechanism also operates in developing wing discs, where overexpression of activated $\mathrm{Ci}$ promotes cell growth by potentiating Yki signaling activity.

In mammals, Glis (a homolog of Drosophila Ci) and YAP (a homolog of Drosophila Yki) have been implicated as proto-oncogenes in many cancers [13, 47, 51, 52]. 
Given that both $\mathrm{Hh}$ and Hippo signaling pathways are highly conserved among species, integration of $\mathrm{Ci}$ into the Yki signaling in this study certainly brings novel insights into our understanding of the mechanistic regulation of the oncogenic Yki/YAP signaling, and potentially provides promising therapeutic strategies for $\mathrm{Hh} / \mathrm{YAP}-\mathrm{re}-$ lated cancer treatment.

\section{Materials and Methods}

\section{Drosophila strains}

Fly stocks used in this study were maintained under standard culture conditions. The w1118 strain was used as the host for all $\mathrm{P}$ element-mediated transformations. Strains carrying $\mathrm{P}\{u a s-y k i\}$, $\mathrm{P}\{u a s-s d\}, \mathrm{P}\{$ uas-hpo $\}, \mathrm{P}\{$ uas-mer $\}, \mathrm{P}\{$ uas-ex $\}, \mathrm{P}\{h s-y k i\} ; y k i^{B 5}$, $\mathrm{P}\{$ uas-wts $\}, \mathrm{P}\{$ uas-vg $\}, \mathrm{P}\{$ actin-Frt-CD2-Frt-gal4 $\} ; \mathrm{P}\{$ diap 1lacZ $\}$, and $\mathrm{P}\{$ actin-Frt-CD2-Frt-gal4 $\} ; \mathrm{P}\{$ ex-lacZ $\}$ were maintained in Shian $\mathrm{Wu}$ lab. Strains $\mathrm{P}\{H h R N A i\}$ and $\mathrm{P}\{$ shmiR-smo $\}$ were purchased from Bloominton. Strains $\mathrm{P}\{$ uas-ykiRNAi\}, $\mathrm{P}\{$ uassdRNAi\}, $\mathrm{P}\{u a s-h p o R N A i\}, \mathrm{P}\{u a s-m e r R N A i\}$, and $\mathrm{P}\{u a s-w t s-$ $R N A i\}$ were purchased from VDRC. Strains $\mathrm{P}\left\{\right.$ uas $\left.-C^{C A}: H A\right\}$, $\mathrm{P}\{c i R N A i\}, \mathrm{P}\{p t c-l a c Z\}$, and $\mathrm{P}\{d p p-l a c Z\}$ were gifts from $\mathrm{Dr}$ Yun Zhao. Strains $\mathrm{P}\{u a s$-tgi $\}, \mathrm{P}\{$ uas-tgiRNAi\}, and $\mathrm{P}\{$ uas-vgR$N A i\}$ were gifts from Dr Lei Zhang. Strains $h h^{A C 2}$ and $h h^{T s}$ mutants were gifts from Dr Yu Cai. Strains $\mathrm{P}\{$ uas $P$-GFP $\}, \mathrm{P}\{$ bamP-GFP $\}$, $\mathrm{P}\{c 587$-gal4 $\}, \mathrm{P}\{$ bab1-gal4 $\}, \mathrm{P}\{$ nos $P$-gal4:vp 16$\}$, and $\mathrm{P}\{A P$ gal4 $\}$ are maintained in Dahua Chen laboratory. Strains $\mathrm{P}\{$ uaspshmiR-yki\} and $\mathrm{P}\{u a s p-s h m i R-c i\}$ were generated in this study. Detailed information regarding the primers used to create these strains can be found in Supplementary information, Data S1.

\section{Flip-Out experiments}

Strains $h s$-flp, actinP-Frt-CD2-Frt-GAL4;ex-lacZ/TM6B or $h s-$ flp, actinP-Frt-CD2-Frt-GAL4;diap1-lacZ/TM6B were crossed with $\mathrm{P}\{u a s P-s h m i R-y k i\} ; \mathrm{P}\left\{u a s-C i^{C A}: H A\right\}$ or $\mathrm{P}\{u a s P-s h m i R-c i\}$. Flys were crossed for 2-3 days before transferring into fresh tubes. The eggs were raised in $25^{\circ} \mathrm{C}$ for $2-3$ days, and first instar larvae were heat-shocked for $15 \mathrm{~min}$ to induce clones. The third instar larvae were dissected for immunohistochemistry.

Strain AP-gal4; $\{$ uas $P$-GFP $\}$ was crossed with $\mathrm{P}\{$ uas- $C i$ $\left.{ }^{C A}: H A\right\}, \mathrm{P}\left\{\right.$ uas $P$-shmiR-yki\}, or $\mathrm{P}\left\{\right.$ uas- $\left.{ }^{C A}{ }^{C A}: H A\right\} ; \mathrm{P}\{$ uas $P$-shmiR$y k i\}$. The third instar larvae were dissected for immunostaining assays. Clones were marked with GFP-positive cells, and the clone sizes were measured using a Zeiss LSM 510 Meta confocal microscope.

\section{Immunohistochemistry for Drosophila ovary}

Ovaries were prepared for immunohistochemistry as described previously [53]. The following primary antibody dilutions were used: rabbit anti-GFP (1:2 000; Invitrogen), mouse anti-Hts (1:1 500; DSHB), rabbit and mouse anti-BamC (1:2 000; [33]), rabbit anti-Vasa (1:2 000; Santa Cruz), rabbit anti-HA(1:2 000; CST), rabbit anti-Hpo (1:500; [36]), rabbit anti-Yki $(1: 1000 ;[19])$, rat anti-Ci (1:2 000; DHSB), and mouse anti- $\beta$-Gal (1:1 000; Promega). The following secondary antibodies were used at a 1:1 500 dilution: goat anti-mouse Alexa568, goat anti-rabbit Alexa488 and goat anti-rat Alexa647 (all from Molecular Probes).

\section{Cell immunofluorescence}

S2 cell immunofluorescence was preformed as described previously [54]. Imaging of these cells was performed using a Zeiss LSM 710 Meta laser-scanning confocal system. The following antibodies were used in this study: rabbit anti-Flag (1:3 000; Sigma) and mouse anti-Myc (1:3 000; MBL). The following secondary antibodies were used at a 1:200 dilution: goat anti-mouse Alexa555 and goat anti-rabbit Alexa488 (Molecular Probes).

\section{Phenotypic analysis}

Ovaries were isolated from 3-day-old flies for immunohistochemistry. Flies for statistics were raised at $29{ }^{\circ} \mathrm{C}$ for 7 days. Images were collected on a Zeiss LSM 710 Meta confocal microscope to count the number of spherical spectrosomes and to identify differentiated cysts with branched fusomes. This protocol was described previously [53]. Three germarium phenotypes, normal-like germaria (number of Sp: 2-7), moderate tumorous germaria (number of Sp: 8-20) and severe tumorous germaria (number of Sp: $>20)$, used for statistic assays were shown in Figure 2A$2 \mathrm{C}$.

Cell culture, immunoprecipitation, and western blot analysis

S2 cells were cultured in Schneider's Drosophila medium (Sigma). Plasmids were transfected into S2 cells. Transfection was performed using the calcium phosphate transfection method. Harvested cells were lysed in the lysis buffer (50 mM Tris $\mathrm{pH}$ $7.5,150 \mathrm{mM} \mathrm{NaCl}, 1 \%$ Triton $\mathrm{X}-100$ and $10 \%$ glycerol). Immunoprecipitation and western blot were performed using protocols described previously [54]. The following reagents were used: rabbit anti-Myc (Santa Cruz), rabbit anti-Flag (Sigma), rabbit anti-Yki, rabbit anti p-Yki (1:5 000; Abmart; [55]), rabbit anti-HA antibodies (Abmart), rabbit anti-HSV (Abcam) and mouse anti-V5 (Immunoway) antibodies, and mouse anti-Flag affinity gel (Sigma).

\section{Preparation of nuclear and cytosolic fractions}

S2 cells were collected by centrifugation, washed twice with $1 \times$ PBS, resuspended with three volumes of hypotonic buffer $(1.5$ $\mathrm{mM} \mathrm{MgCl} 2,10 \mathrm{mM} \mathrm{KCl}, 10 \mathrm{mM}$ HEPES ( $\mathrm{pH} 7.5)$, and protease inhibitor cocktail (1:100; Roche)) and incubated on ice for 20 min. The resuspended cells were transferred into a 1-ml Dounce homogenizer and homogenized with tight strokes 100 times. The homogenates were then centrifuged at $1000 \times g$ at $4{ }^{\circ} \mathrm{C}$. The supernatant was collected as the cytosolic fraction, and the pellet was washed twice with hypotonic buffer and collected as the nuclear fraction. Nuclei were collected as described above, and then lysed in the lysis buffer (0.2 mM EGTA, $3 \mathrm{mM}$ EDTA, and protease inhibitor cocktail (1:100; Roche)) for $20 \mathrm{~min}$.

\section{Quantitative RT-PCR}

Total RNA was isolated with Trizol Reagent (Tiangen) and cDNA was synthesized using FastQuant RT Kit (With gDNase, Tiangen). Quantitative RT-PCR was performed using UltraSYBR mixture (cwbiotech) in triplicate on LightCycler 480 (Roche). Template concentrations were normalized to endogenous reference rp49. Primers for quantitative RT-PCR are shown in Supplementary information, Table $\mathrm{S} 1$.

Primers for Arti-miR-knockdown flies 
$\mathrm{P}\{$ uasp-shmiR-yki\}:5'-ctagcagtGCTATTCGACTGCGTCCTGTAtagttatattcaagcataTTCAGGACGCTGTCGAATAGCgcg-3'

5'-aattcgcGCTATTCGACAGCGTCCTGAAtatgettgaatataactaTACAGGACGCAGTCGAATAGCactg-3'

5'-ctagcagtGGAGCAAGCCTAGACCAATCAtagttatattcaagcataTCATTGGTCTTGGCTTGCTCCgcg-3'

5'-aattcgcGGAGCAAGCCAAGACCAATGAtatgettgaatataactaTGATTGGTCTAGGCTTGCTCCactg-3'

$\mathrm{P}\{$ uasp-shmiR-ci\}:5'-ctagcagtCGGAGATCATCATAATGAATTtagttatattcaagcataATTTCATTATCATGATCTCCGgcg-3'

5'-aattcgcCGGAGATCATGATAATGAAATtatgettgaatataactaAATTCATTATGATGATCTCCGactg-3'

5'-ctagcagtGCAAGATGAAGTTGTTAAAGAtagttatattcaagcataTGTTTAACAAGTTCATCTTGCgcg-3'

5'-aattcgcGCAAGATGAACTTGTTAAACAtatgettgaatataactaTCTTTAACAACTTCATCTTGCactg-3'

\section{Acknowledgments}

This work was supported by the National Basic Research Program of China (2013CB945000 and 2011CB943903), the Strategic Priority Research Program of the Chinese Academy of Sciences (XDA01010306), and the National Natural Science Foundation of China (91019022, 31130036, 31329004, and 31171411).

\section{References}

1 Fuchs E, Tumbar T, Guasch G. Socializing with the neighbors: stem cells and their niche. Cell 2004; 116:769-778.

2 Spradling A, Fuller MT, Braun RE, Yoshida S. Germline stem cells. Cold Spring Harb Perspect Biol 2011; 3:a002642.

3 Kirilly D, Xie T. The Drosophila ovary: an active stem cell community. Cell Res 2007; 17:15-25.

4 Xie T, Spradling AC. A niche maintaining germ line stem cells in the Drosophila ovary. Science 2000; 290:328-330.

5 Kirilly D, Wang S, Xie T. Self-maintained escort cells form a germline stem cell differentiation niche. Development 2011; 138:5087-5097.

6 Decotto E, Spradling AC. The Drosophila ovarian and testis stem cell niches: similar somatic stem cells and signals. Dev Cell 2005; 9:501-510.

7 Chen D, McKearin D. Dpp signaling silences bam transcription directly to establish asymmetric divisions of germline stem cells. Curr Biol 2003; 13:1786-1791.

8 Chen D, McKearin DM. A discrete transcriptional silencer in the bam gene determines asymmetric division of the Drosophila germline stem cell. Development 2003; 130:1159-1170.

9 Xie T, Spradling AC. decapentaplegic is essential for the maintenance and division of germline stem cells in the Drosophila ovary. Cell 1998; 94:251-260.

10 Song X, Wong MD, Kawase E, et al. Bmp signals from niche cells directly repress transcription of a differentiation-promoting gene, bag of marbles, in germline stem cells in the Drosophila ovary. Development 2004; 131:1353-1364.

11 Xia L, Zheng X, Zheng W, et al. The niche-dependent feedback loop generates a BMP activity gradient to determine the germline stem cell fate. Curr Biol 2012; 22:515-521.

12 Xia L, Jia S, Huang S, et al. The Fused/Smurf complex con- trols the fate of Drosophila germline stem cells by generating a gradient BMP response. Cell 2010; 143:978-990.

13 Pan D. The hippo signaling pathway in development and cancer. Dev Cell 2010; 19:491-505.

14 Edgar BA. From cell structure to transcription: hippo forges a new path. Cell 2006; 124:267-273.

15 Halder G, Johnson RL. Hippo signaling: growth control and beyond. Development 2011; 138:9-22.

16 Staley BK, Irvine KD. Hippo signaling in Drosophila: recent advances and insights. Dev Dyn 2012; 241:3-15.

17 Harvey K, Tapon N. The Salvador-Warts-Hippo pathway - an emerging tumour-suppressor network. Nat Rev Cancer 2007; 7:182-191.

18 Yu FX, Guan KL. The Hippo pathway: regulators and regulations. Genes Dev 2013; 27:355-371.

19 Huang J, Wu S, Barrera J, Matthews K, Pan D. The Hippo signaling pathway coordinately regulates cell proliferation and apoptosis by inactivating Yorkie, the Drosophila Homo$\log$ of YAP. Cell 2005; 122:421-434.

20 Ramos A, Camargo FD. The Hippo signaling pathway and stem cell biology. Trends Cell Biol 2012; 22:339-346.

21 Wu S, Liu Y, Zheng Y, Dong J, Pan D. The TEAD/TEF family protein Scalloped mediates transcriptional output of the Hippo growth-regulatory pathway. Dev Cell 2008; 14:388-398.

22 Zhang L, Ren F, Zhang Q, Chen Y, Wang B, Jiang J. The TEAD/TEF family of transcription factor Scalloped mediates Hippo signaling in organ size control. Dev Cell 2008; 14:377387.

23 Goulev Y, Fauny JD, Gonzalez-Marti B, Flagiello D, Silber J, Zider A. SCALLOPED interacts with YORKIE, the nuclear effector of the hippo tumor-suppressor pathway in Drosophila. Curr Biol 2008; 18:435-441.

24 Koontz LM, Liu-Chittenden Y, Yin F, et al. The hippo effector yorkie controls normal tissue growth by antagonizing scalloped-mediated default repression. Dev Cell 2013; 25:388401.

25 Guo T, Lu Y, Li P, et al. A novel partner of Scalloped regulates Hippo signaling via antagonizing Scalloped-Yorkie activity. Cell Res 2013; 23:1201-1214.

26 Attisano L, Wrana JL. Signal integration in TGF-beta, WNT, and Hippo pathways. F1000Prime Rep 2013; 5:17.

27 Guo X, Wang XF. Signaling cross-talk between TGF-beta/ BMP and other pathways. Cell Res 2009; 19:71-88.

28 King FJ, Szakmary A, Cox DN, Lin H. Yb modulates the divisions of both germline and somatic stem cells through piwiand hh-mediated mechanisms in the Drosophila ovary. Mol Cell 2001; 7:497-508.

29 Shcherbata HR, Ward EJ, Fischer KA, et al. Stage-specific differences in the requirements for germline stem cell maintenance in the Drosophila ovary. Cell Stem Cell 2007; 1:698709.

30 Sun S, Zhao S, Wang Z. Genes of Hippo signaling network act unconventionally in the control of germline proliferation in Drosophila. Dev Dyn 2008; 237:270-275.

31 Wang H, Mu Y, Chen D. Effective gene silencing in Drosophila ovarian germline by artificial microRNAs. Cell Res 2011; 21:700-703.

32 Kai T, Spradling A. An empty Drosophila stem cell niche reactivates the proliferation of ectopic cells. Proc Natl Acad Sci 
USA 2003; 100:4633-4638.

33 Chen D, Wang Q, Huang H, Xia L, Jiang X, et al. Effete-mediated degradation of Cyclin A is essential for the maintenance of germline stem cells in Drosophila. Development 2009; 136:4133-4142.

34 Copps K, Richman R, Lyman LM, Chang KA, Rampersad-Ammons J, Kuroda MI. Complex formation by the Drosophila MSL proteins: role of the MSL2 RING finger in protein complex assembly. EMBO J 1998; 17:5409-5417.

35 Zhang Y, Malone JH, Powell SK, et al. Expression in aneuploid Drosophila S2 cells. PLoS Biol 2010; 8:e1000320.

36 Wu S, Huang J, Dong J, Pan D. hippo encodes a Ste-20 family protein kinase that restricts cell proliferation and promotes apoptosis in conjunction with salvador and warts. Cell 2003; 114:445-456.

37 Chen HJ, Wang CM, Wang TW, et al. The Hippo pathway controls polar cell fate through Notch signaling during Drosophila oogenesis. Dev Biol 2011; 357:370-379.

38 Forbes AJ, Lin H, Ingham PW, Spradling AC. Hedgehog is required for the proliferation and specification of ovarian somatic cells prior to egg chamber formation in Drosophila. Development 1996; 122:1125-1135.

39 Ingham PW, McMahon AP. Hedgehog signaling in animal development: paradigms and principles. Genes Dev 2001; 15:3059-3087.

40 Ingham PW, Nakano Y, Seger C. Mechanisms and functions of Hedgehog signalling across the metazoa. Nat Rev Genet 2011; 12:393-406.

41 Zhang Y, Kalderon D. Hedgehog acts as a somatic stem cell factor in the Drosophila ovary. Nature 2001; 410:599-604.

42 Konig A, Shcherbata HR. Soma influences GSC progeny differentiation via the cell adhesion-mediated steroid-let-7Wingless signaling cascade that regulates chromatin dynamics. Biol Open 2015; 4:285-300.

43 Hamada-Kawaguchi N, Nore BF, Kuwada Y, Smith CI, Yamamoto D. Btk29A promotes Wnt4 signaling in the niche to terminate germ cell proliferation in Drosophila. Science 2014; 343:294-297.

44 Maimon I, Popliker M, Gilboa L. Without children is required for Stat-mediated zfh1 transcription and for germline stem cell differentiation. Development 2014; 141:2602-2610.

45 Chau J, Kulnane LS, Salz HK. Sex-lethal facilitates the transition from germline stem cell to committed daughter cell in the Drosophila ovary. Genetics 2009; 182:121-132.

46 Tastan OY, Maines JZ, Li Y, McKearin DM, Buszczak M. Drosophila ataxin 2-binding protein 1 marks an intermediate step in the molecular differentiation of female germline cysts. Development 2010; 137:3167-3176.

47 Zhao B, Li L, Lei Q, Guan KL. The Hippo-YAP pathway in organ size control and tumorigenesis: an updated version. Genes Dev 2010; 24:862-874.

48 Rojas-Rios P, Guerrero I, Gonzalez-Reyes A. Cytoneme-mediated delivery of hedgehog regulates the expression of bone morphogenetic proteins to maintain germline stem cells in Drosophila. PLoS Biol 2012; 10:e1001298.

49 Amoyel M, Simons BD, Bach EA. Neutral competition of stem cells is skewed by proliferative changes downstream of Hh and Hpo. EMBO J 2014; 3:2295-313.

50 Huang J, Kalderon D. Coupling of Hedgehog and Hippo pathways promotes stem cell maintenance by stimulating proliferation. J Cell Biol 2014; 205:325-338.

51 Dahmane N, Sanchez P, Gitton Y, et al. The Sonic Hedgehog-Gli pathway regulates dorsal brain growth and tumorigenesis. Development 2001; 128:5201-5212.

52 Clement V, Sanchez P, de Tribolet N, Radovanovic I, Ruiz i Altaba A. HEDGEHOG-GLI1 signaling regulates human glioma growth, cancer stem cell self-renewal, and tumorigenicity. Curr Biol 2007; 17:165-172.

53 Yang L, Chen D, Duan R, et al. Argonaute 1 regulates the fate of germline stem cells in Drosophila. Development 2007; 134:4265-4272.

54 Ji S, Sun M, Zheng X, et al. Cell-surface localization of Pellino antagonizes Toll-mediated innate immune signalling by controlling MyD88 turnover in Drosophila. Nat Commun 2014; 5:3458.

55 Dong J, Feldmann G, Huang J, et al. Elucidation of a universal size-control mechanism in Drosophila and mammals. Cell 2007; 130:1120-1133.

(Supplementary information is linked to the online version of the paper on the Cell Research website.) 